\title{
4D large scale variational data assimilation of a turbulent flow with a dynamics error model
}

\author{
Pranav Chandramouli ${ }^{1}$, Etienne Memin \\ Fluminance - INRIA, 263 Avenue General Leclerc, Rennes \\ Dominique Heitz \\ Irstea, UR OPAALE, F-35044 Rennes Cedex, France
}

\begin{abstract}
We present a variational assimilation technique (4D-Var) to reconstruct time resolved incompressible turbulent flows from measurements on two orthogonal $2 \mathrm{D}$ planes. The proposed technique incorporates an error term associated to the flow dynamics. It is therefore a compromise between a strong constraint assimilation procedure (for which the dynamical model is assumed to be perfectly known) and a weak constraint variational assimilation which considers a model enriched by an additive Gaussian forcing. The first solution would require either an unaffordable direct numerical simulation (DNS) of the model at the finest scale or an inaccurate and numerically unstable large scale simulation without parametrisation of the unresolved scales. The second option, the weakly constrained assimilation, relies on a blind error model that needs to be estimated from the data. This latter option is also computationally impractical for turbulent flow models as it requires to augment the state variable by an error variable of the same dimension. The proposed 4D-Var algorithm is successfully applied on a $3 \mathrm{D}$ turbulent wake flow in the transitional regime without specifying the obstacle geometry. The algorithm is validated on a synthetic 3D data-set with full-scale information. The performance of the algorithm is further analysed on data emulating large-scale experimental PIV observations.
\end{abstract}

\footnotetext{
${ }^{1}$ Corresponding author; Email: pranav.chandramouli@inria.fr
} 
Keywords: 4D variation assimilation, turbulent wake flow, adjoint-optimisation, stochastic flow dynamics, dynamics error model

\section{Introduction}

Computational fluid dynamics (CFD) and experimental fluid dynamics (EFD) represent two established yet limited sets of techniques for the study of fluid flows. Both family of methods provides distinct advantages in representing and 5 studying fluid flows. Similarly, they are both restricted in application by certain limitations. These limitations display a complementarity between the two fields. CFD is limited by the accuracy of its inlet and initial conditions, while EFD is capable of measuring an accurate initial and inlet conditions. The latter is limited in spatial extent while a large domain can be simulated using the former.

EFD is capable of measuring accurate but sparse and selective flow field properties while CFD is capable of measuring an approximate but complete flow-field properties over a large domain. The exploitation of this complementarity by using a dynamical model guided by experimental observations is termed as data assimilation (DA).

DA, as a field, has been mainly driven by the works of researchers from the atmospheric and oceanographic sciences. The existing set of DA procedures can be broadly categorised into two main categories. Methods which are derived from stochastic filtering principles fall under the category of sequential data assimilation (SDA) approaches. The particle filter method [19], the Ensemble

20 Kalman Filter (EnKF) [13, or a combination of both methods 33] are prominent examples of this method. These Monte Carlo methods are generally based on the principle of Bayesian minimum variance estimation. They are termed as 'sequential' due to the constant forward propagation of the system state statistics. Observations are assimilated to correct the predicted state when measurements are available along the state trajectory.

The second category of DA procedures are referred to as variational data assimilation (VDA) approaches and these originate from concepts of optimal con- 
trol theory and variation calculus thus deriving the name [27]. These methods aim to estimate the optimal trajectory, starting from a background condition, which minimises a cost function leading to lowest error between system and observations. The works of Bergthórsson and Döös [2], Cressman [10] on optimal interpolation methods were stepping stones to the VDA methods. With VDA, the approaches can be classified into $3 \mathrm{D}$ variational and $4 \mathrm{D}$ variational methods depending on the spatial dimensions of the simulation and the inclusion, or not, 35 of a temporal window for the system's dynamical evolution. A first application of such methods was done by Sasaki [38] who further extended them to 4D analysis in Sasaki 39. Since then, variants of the VDA approach have been used for DA with Le Dimet and Talagrand [26] being the prominent seminal reference for large scale data driven forecasting issues related to geophysical fluids.

40 Both categories of DA methods require significant computational power in order to provide meaningful results. This has constrained the application of DA to simple 2D flows in fluid mechanics such as the works of Mons et al. [30, 31]. In the context of SDA methods, Meldi and Poux [28] proposed a Kalman filter based assimilation technique for the reconstruction of 3D, un45 steady turbulent flows using a reduced order model for cost-reduction. The successful application of the method portrayed the capability of DA methods despite the sub-optimality of Kalman filters in a nonlinear context. Astutely, a reduced order expression of the error covariance matrix, is introduced and aims at the same state-space reduction objective as those achieved by ensemble

50 Kalman procedures (EnKF) [14]. However, contrary to those Monte-Carlo filters the nonlinearity of the dynamics is not fully taken into account. Furthermore, the turbulent "error" model is not optimised through the assimilation. Given the varied strengths of the two DA methods, VDA is better suited for state estimation while SDA has been the optimal choice for parameter estimation.

55 This is because VDA takes into account future observations for state estimation while SDA corrects the current state of the system based only on current and past observations. On the other hand, it is easier to perform parameter optimisation in SDA as in VDA parameter optimisation requires the adjoint of the 
dynamical model with respect to the parameter. However, this was observed to be challenging to perform even using SDA unless in a reduced-order sense by 43 .

This work is a first attempt, to the extent of the Authors' knowledge, at simulating 3D, unsteady turbulent flows using principles of VDA from an experimentally realisable set of PIV data. The VDA approach developed by Gronskis et al. 20] for 2D DNS of cylinder wake flow forms a basis for this work. A similar adjoint-based approach using PTV data was done by 40, for a planar jet. The ideal $4 \mathrm{D}$ assimilation with a perfect dynamical model would require an impractical DNS which is clearly unaffordable. In the proposed method, the computational cost reduction is achieved by coupling a recently proposed flow

70 model, arising from a stochastic analysis of error propagation, termed modelling under location uncertainty (LU) by Mémin [29] (described in \$3) with the assimilation algorithm. This strategy enables a significant reduction in the resolution required for the simulation and provides a meaningful error or "turbulence" model associated to the unresolved component of the flow. In addition, such 75 a coupling provides an opportunity to tune the contribution of the model by introducing it as a control parameter in the optimisation procedure. Thus, the methodology used for cost-reduction, which tends to introduce errors, is itself corrected by the assimilation algorithm. The ability to locally optimise model contribution is an important research question in the field of DA - this is explored in $\S 5$. This technique enables an alternative solution to the blind weakly constrained assimilation technique in which an error variable is added to the unknown state variable (namely the velocity and pressure). In such weakly constrained system, the error variable is generally modelled as an additive forcing variable varying in space and time - and thus of the same dimension as the state variable. The corresponding control problem, which requires the estimation of an initial condition, an inlet and/or outlet condition and a full trajectory of an error variable related to the unresolved small scales, is computationally very expensive. Such a solution is in practice restricted to the assimilation of reduced order models [1, 11. Contrary to this unaffordable solution, the technique we 
propose, relies on the estimation of a stationary spatially varying coefficient of an adapted error model that accounts for unresolved turbulent scales of motion.

The principles of VDA and the adjoint-based optimisation procedure is enumerated in $\$ 2$. The formulation of the LU model and its numerical treatment for VDA is presented in $\$ 3$. The resultant code, termed as 4D-Var, is capable of performing VDA on flows of higher Reynolds numbers that were previously limited by high computational cost. This $4 \mathrm{D}$-Var approach is used to optimise a three dimensional three component (3D3C) velocity field for wake flow at $R e=3900$ while assimilating time-resolved observations in 4 . The possibility to tune the error model by introducing the model coefficient as a control parameter in the optimisation algorithm is explored in $\$ 5$ A final section of concluding remarks follows.

\section{Variational data assimilation}

\subsection{Mathematical representation:}

DA techniques have a dual objective: to improve knowledge of the current system trajectory $\left(\mathcal{X}_{t}\right)$ (also called the analysis trajectory) based on observations $\left(\mathcal{Y}_{t}\right)$ and an a priori known background condition $\left(\mathcal{X}^{b}\right)$, and to predict an accurate future state of the system from current and past observations. Mathematically it can be expressed as:

$$
\begin{array}{r}
\partial_{t} \mathcal{X}_{t}(\mathbf{x})+\mathbb{M}\left(\mathcal{X}_{t}(\mathbf{x})\right)=\mathbf{q}_{t} \\
\mathcal{X}_{0}(\mathbf{x})=\mathcal{X}_{0}^{b}(\mathbf{x})+\boldsymbol{\eta}, \\
\mathcal{Y}_{t}=\mathbb{H}\left(\mathcal{X}_{t}(\mathbf{x})\right)+\boldsymbol{\epsilon}_{t}
\end{array}
$$

where the state space trajectory $\mathcal{X}_{t}$ is provided through the integration of a dynamical evolution model $\mathbb{M}$ of the system from an initial condition a priori known only up to a noisy background state $\mathcal{X}_{0}^{b}$ and, from a sparse set of noisy observations $\mathcal{Y}$. The sub-script denotes the temporal state of the system, and the super-script denotes the type of system state, i.e. background (b). 
The noise attached to the dynamics, the initial condition and the observations (respectively $\mathbf{q}, \boldsymbol{\eta}$, and $\boldsymbol{\epsilon}$ ) are Gaussian variables.

The temporal evolution of the state in space $(\mathbf{x})$ and time $(t)$ through the dynamical model $\mathbb{M}$ is denoted by eq. (1) up to a model error $\mathbf{q}_{t}$. In the context of this work, the state of the system denotes the velocity and pressure fields while the dynamical model is the NS equation given as,

$$
\frac{\partial \boldsymbol{u}}{\partial t}+\boldsymbol{u} \cdot \nabla \boldsymbol{u}=-\frac{1}{\rho} \nabla p+\nu \Delta \boldsymbol{u}
$$

where $\boldsymbol{u}$ is the velocity field, $p$, the pressure, $\rho$, the fluid density, and $\nu$ stands for molecular viscosity. The model error is assumed to take the form of a centered Gaussian random variable with covariance $\boldsymbol{Q}$.

The second equation equates the state of the system at time $t=0$, i.e. $\mathcal{X}_{0}(\mathbf{x})$ to the a priori known background state $\mathcal{X}_{0}^{b}(\mathbf{x})$ up to an error $\boldsymbol{\eta}(\mathbf{x})$. The background error is assumed to be of zero mean and associated to the background covariance matrix $\boldsymbol{B}$ as,

$$
\boldsymbol{B}=\mathbb{E}\left(\left(\boldsymbol{\mathcal { X }}_{0}^{b}-\mathcal{X}_{0}\right)\left(\mathcal{X}_{0}^{b}-\mathcal{X}_{0}\right)^{T}\right)=\mathbb{E}\left(\boldsymbol{\eta} \boldsymbol{\eta}^{T}\right)
$$

The definition of this background covariance matrix is of significance in DA.

115 A method for defining this background covariance matrix using singular value decomposition (SVD) techniques is explored in 4.4.3.

The final equation relates the observations $\mathcal{Y}$ with the state variable $\mathcal{X}$ through the observation operator $\mathbb{H}$ which can be non-linear. This model is assumed to be accurate up to an observation error $\boldsymbol{\epsilon}(t, \mathbf{x})$. This error arises due to sparseness of the observations, or due to scale dissimilarity between the observations and the state space or due to a noisy set of observations arising from experimental limitations. It covers equipment errors as well as errors arising from the observation operator. The error is assumed to be a zero mean Gaussian random field with the associated covariance tensor $\boldsymbol{R}$. All these Gaussian assumptions on the different noises involved yields a joint probability distribution that can be expressed through its logarithm as a cost functional. 


\subsection{Cost Functional}

The dynamical system is controlled by an initial condition and a forcing error term, which is a function of time and space. The cost function $J\left(\boldsymbol{\eta}, \mathbf{q}_{t}\right)$ associated to these two unknown quantities can be expressed as:

$$
\begin{array}{r}
J\left(\boldsymbol{\eta}, \mathbf{q}_{t}\right)=\frac{1}{2}\left(\mathcal{X}_{0}-\mathcal{X}_{0}^{b}\right)^{T} \boldsymbol{B}^{-1}\left(\mathcal{X}_{0}-\mathcal{X}_{0}^{b}\right)+\frac{1}{2} \int_{t_{0}}^{t_{f}}\left(\mathbb{H}\left(\mathcal{X}_{t}\right)-\mathcal{Y}_{t}\right)^{T} \boldsymbol{R}^{-1}\left(\mathbb{H}\left(\mathcal{X}_{t}\right)-\mathcal{Y}_{t}\right) \\
+\frac{1}{2} \int_{t_{0}}^{t_{f}} \mathbf{q}_{t}^{T} \boldsymbol{Q}^{-1} \mathbf{q}_{t},
\end{array}
$$

where $t_{0}$ and $t_{f}$ are initial and final time of the assimilation window and the evolution of the state of the system $\boldsymbol{\mathcal { X }}_{t}$ is given formally as,

$$
\mathcal{X}_{t}=\mathcal{X}_{0}+\int_{t_{0}}^{t} \mathbb{M}\left(\mathcal{X}_{s}\right) d s+\int_{t_{0}}^{t} \mathbf{q}_{s} d s=\psi_{t}\left(\mathcal{X}_{0}, \mathbf{q}_{t}\right),
$$

where $\psi_{t}\left(\boldsymbol{\mathcal { X }}_{0}, \mathbf{q}_{t}\right)$ is the flow map, which depends on the initial condition but also on the forcing along time.

The first term in the cost function accounts for the error between the initial condition $\mathcal{X}_{0}$ and the a priori known background condition $\mathcal{X}_{0}^{b}$ weighted by the inverse of the background error covariance matrix $\boldsymbol{B}^{-1}$. The second term is the error between the state trajectory $\mathcal{X}_{t}$ as obtained using the dynamical model $(\mathbb{M})$ and the observations $(\mathcal{Y})$ using an appropriate observation operator $(\mathbb{H})$ and weighted by the inverse of the observation error covariance matrix $\boldsymbol{R}^{-1}$. The third term corresponds to the norm of the error forcing term weighted by the covariance matrix for the error model. Before entering into the details on why such a formulation is a bad idea for our application, we need to describe briefly in the following, the way the optimisation of such a functional can be performed in practice.

\subsection{Adjoint method}

To optimise the cost functional, a gradient descent methodology needs to be applied, which requires the explicit calculation of the gradient. Classical methods such as finite difference cannot be employed here - for DA studies of fluid 
integrations of the dynamics for each component of the initial condition are totally excluded. An elegant solution to do this at a reduced cost is the adjoint method, seminally proposed by Lions [27] and applied to the DA context by Le Dimet and Talagrand [26].

The adjoint model computes the gradient of the cost function in the direction $\delta \mathbf{n}=\left(\delta \mathbf{q}_{t}, \delta \boldsymbol{\eta}\right)$ with a single integration of the adjoint of the tangent linear dynamical model backwards in time. Consider an adjoint variable $\boldsymbol{\lambda}$ belonging to the same state space and integrated over the time range of the assimilation, the inner product of this variable with tangent linear model of the non-linear dynamical model gives,

$$
\int_{t_{0}}^{t_{f}}\left\langle\frac{\partial d \mathcal{X}_{t}}{\partial t}, \boldsymbol{\lambda}_{t}\right\rangle d t+\int_{t_{0}}^{t_{f}}\left\langle\partial_{\mathcal{X}} \mathbb{M} d \mathcal{X}_{t}, \boldsymbol{\lambda}_{t}\right\rangle d t=\int_{t_{0}}^{t_{f}}\left\langle\delta \mathbf{q}_{t}, \boldsymbol{\lambda}_{t}\right\rangle d t
$$

where,

$$
\begin{aligned}
\partial_{t} d \mathcal{X}(t, \mathbf{x})+\partial_{\mathcal{X}} \mathbb{M}(\mathcal{X}(t, \mathbf{x})) d \mathcal{X}(t, \mathbf{x}) & =\delta \mathbf{q}_{t}(\mathbf{x}), \\
d \mathcal{X}\left(t_{0}, \mathbf{x}\right) & =\delta \boldsymbol{\eta}(\mathbf{x}),
\end{aligned}
$$

is the linear evolution model of the differential $d \mathcal{X}=\partial_{\mathbf{q}} \mathbb{M} \delta \mathbf{q}_{t}+\partial_{\boldsymbol{\eta}} \mathbb{M} \delta \boldsymbol{\eta}$, with $\partial_{\mathcal{X}} \mathbb{M}$ denoting the tangent linear operator associated to the non-linear model operator $\mathbb{M}$, and $\langle.,$.$\rangle stands for the \mathbb{L}^{2}$ inner product. The corresponding gradient of the tangent linear model along a direction $\delta \mathbf{n}$ is,

$$
\begin{array}{r}
\left\langle\frac{\partial J}{\partial \mathbf{n}}, \delta \mathbf{n}\right\rangle=\left\langle\boldsymbol{B}^{-1}\left(\mathcal{X}_{0}-\mathcal{X}_{0}^{b}\right), \delta \boldsymbol{\eta}\right\rangle-\int_{t_{0}}^{t_{f}}\left\langle\left(\partial_{\mathcal{X}} \mathbb{H}\right)^{*} \boldsymbol{R}^{-1}\left(\mathcal{Y}_{t}-\mathbb{H}\left(\mathcal{X}_{t}\right)\right), \frac{\partial \mathcal{X}}{\partial \mathbf{n}} \delta \mathbf{n}\right\rangle d t \\
+\int_{t_{0}}^{t_{f}}\left\langle\mathbf{Q}^{-1}\left(\partial_{t} \mathcal{X}+\mathbb{M}\left(\mathcal{X}_{t}\right)\right), \delta \mathbf{q}_{t}\right\rangle d t
\end{array}
$$

where the adjoint operator of the for the linearised observation operator is introduced,

$$
\left\langle\left(\partial_{\mathcal{X}} \mathbb{H}(\mathcal{X})\right) \mathbf{f}, \mathbf{g}\right\rangle=\left\langle\mathbf{f},\left(\partial_{\mathcal{X}} \mathbb{H}(\mathcal{X})\right)^{*} \mathbf{g}\right\rangle
$$

Applying integration by parts to the right-hand side of (8) gives,

$-\int_{t_{0}}^{t_{f}}\left\langle-\frac{\partial \boldsymbol{\lambda}_{t}}{\partial t}+(\partial \mathcal{X} \mathbb{M})^{*} \boldsymbol{\lambda}_{t}, d \boldsymbol{\mathcal { X }}_{t}\right\rangle d t=\left\langle\boldsymbol{\lambda}_{t_{f}}, d \mathcal{X}_{t_{f}}\right\rangle-\left\langle\boldsymbol{\lambda}_{t_{0}}, d \boldsymbol{\mathcal { X }}_{t_{0}}\right\rangle+\int_{t_{0}}^{t_{f}}\left\langle\delta \mathbf{q}_{t}, \boldsymbol{\lambda}_{t}\right\rangle d t$ 
where $\left(\partial_{\mathcal{X}} \mathbb{M}\right)^{*}$ denotes the adjoint of the dynamics model tangent linear operator $\left(\partial_{\mathcal{X}} \mathbb{M}\right)$. We impose the adjoint variable to be a solution of the following adjoint equation system:

$$
\left\{\begin{array}{l}
-\partial_{t} \boldsymbol{\lambda}_{t}+\left(\partial_{\mathcal{X}} \mathbb{M}\right)^{*} \boldsymbol{\lambda}_{t}=\left(\partial_{\mathcal{X}} \mathbb{H}\right)^{*} \boldsymbol{R}^{-1}\left(\mathcal{Y}-\mathbb{H}\left(\mathcal{X}_{t}\right)\right), \\
\boldsymbol{\lambda}_{t_{f}}=0
\end{array}\right.
$$

We can now get the gradient of the cost function by inserting this relation in eq. 12 and equating $d \boldsymbol{\mathcal { X }}_{t_{0}}=\delta \boldsymbol{\eta}$ and $d \boldsymbol{X}=(\partial \mathcal{X} / \partial \mathbf{n}) \delta \mathbf{n}$ as,

$$
\begin{gathered}
\frac{\partial J}{\partial \boldsymbol{\eta}}=-\boldsymbol{\lambda}_{t_{0}}+\boldsymbol{B}^{-1}\left(\delta \boldsymbol{\mathcal { X }}_{0}^{b}-\delta \boldsymbol{\mathcal { X }}_{0}\right), \\
\frac{\partial J}{\partial \mathbf{q}}=\mathbf{Q}^{-1}\left(\partial_{t} \boldsymbol{\mathcal { X }}+\mathbb{M}\left(\boldsymbol{\mathcal { X }}_{t}\right)\right)-\boldsymbol{\lambda} .
\end{gathered}
$$

To obtain the gradient of the cost function, a solution of the (backward) adjoint equation is necessary. However, through (15) we see that the knowledge of the whole adjoint variable must be computed and stored to get the gradient with respect to the error. Furthermore simulations of the forced dynamical system must be performed to evaluate the right-hand side of (13). The simulation of these two inter-dependant equations have to be performed until convergence.

Beyond the tricky character of this inter-dependance, in our case, at a more fundamental standpoint, the model error represents the error of the unresolved flow components whose action is nevertheless visible in the observations. They are associated to the fine scale flow structures and to turbulence. Due to the assumption introduced in this weakly constrained assimilation procedure, this turbulent component is represented by a Gaussian forcing variable, which is known to be a very restrictive assumption for turbulence modelling. A section of established turbulence models are based on a quasi-Gaussian hypothesis for turbulence closure. However, this is different from introducing directly a Gaussian forcing in data assimilation. The latter assumes that the contribution of the SGS terms is a Gaussian force which is a restrictive assumption. In addition, such a Gaussian forcing introduces additional energy in the system which is, in this case, not dissipated due to a lack of an additional dissipation term. In 
the former case, Gaussian closure is used as a methodology to close the pdf of turbulence statistics. It is not achieved by adding a Gaussian variable directly in the equation. If we do apply a quasi-Gaussian hypothesis for turbulence closure and introduce such a model with the associated dissipation term, this would be along the same lines as this work albeit with a different model whose applicability will have to be analysed.

Given these restrictions, an alternative outlook is necessary to proceed further. Instead of a "blind" non-informative external Gaussian forcing, the error is now introduced directly in the model dynamics operator by replacing eq. (1) with:

$$
\partial_{t} \mathcal{X}+\mathbb{M}\left(\mathcal{X}, \mathbf{q}_{t}\right)=0
$$

In this system, we directly introduce the effect of the error on the dynamics through a function of the model error. We will see in section 3 how this can be precisely done for fluid dynamics through a stochastic framework that takes explicitly into account these errors. As a consequence, we revert back to a strong constraint assimilation problem. In its simplest expression, for a known characterisation of the error function, only the initial condition is an unknown variable, and we get back to a simplified functional of the form :

$J(\boldsymbol{\eta})=\frac{1}{2}\left(\mathcal{X}_{0}-\mathcal{X}_{0}^{b}\right)^{T} \boldsymbol{B}^{-1}\left(\mathcal{X}_{0}-\mathcal{X}_{0}^{b}\right)+\frac{1}{2} \int_{t_{0}}^{t_{f}}\left(\mathbb{H}\left(\mathcal{X}_{t}\right)-\mathcal{Y}_{t}\right)^{T} \boldsymbol{R}^{-1}\left(\mathbb{H}\left(\mathcal{X}_{t}\right)-\mathcal{Y}_{t}\right)$

whose gradiant with respect to the initial condition is given by:

$$
\frac{\partial J}{\partial \boldsymbol{\eta}}=-\boldsymbol{\lambda}_{t_{0}}+\boldsymbol{B}^{-1}\left(\delta \boldsymbol{\mathcal { X }}_{0}^{b}-\delta \mathcal{X}_{0}\right)
$$

The gain in using the strong constraint procedure comes from two sources: firstly, the forcing in the weak procedure, that needs to be estimated at each time-step, is no longer explicit but integrated into the dynamical model, and secondly, the coupling of the inter-dependant equations $\sqrt{13}$ ) and $(15)$ is eliminated as the error is not a state variable in the strong-constraint procedure. This error can be optimised by introducing additional control variables in the assimilation but this is an optional control parameter. 


\subsection{Additional control}

When the error function is a priori not known, which is generally the case in practice, an additional control on the error function parameters $\boldsymbol{\vartheta}_{t}$ can be introduced. Other parameters of the dynamical system related for instance to unknown inlet flow conditions can also be introduced through these additional control parameters. The dynamical model is now:

$$
\partial_{t} \mathcal{X}_{t}+\mathbb{M}\left(\mathcal{X}_{t}, \vartheta_{t}\right)=0
$$

The new associated cost function, introduces a penalisation term on the error function,

$J(\boldsymbol{\eta}, \boldsymbol{\vartheta})=\frac{1}{2}\left\|\boldsymbol{\mathcal { X }}_{0}^{b}-\boldsymbol{\mathcal { X }}_{0}\right\|_{\boldsymbol{B}^{-1}}^{2}+\frac{1}{2} \int_{t_{0}}^{t_{f}}\left\|\mathcal{Y}_{t}-\mathbb{H}\left(\boldsymbol{\mathcal { X }}_{t}\right)\right\|_{\boldsymbol{R}^{-1}}^{2} d t+\frac{1}{2} \int_{t_{0}}^{t_{f}}\left\|\boldsymbol{\vartheta}_{t}-\boldsymbol{\vartheta}_{t}^{c}\right\|_{\boldsymbol{B}_{c}^{-1}}^{2} d t$

The cost function now has to minimise, alongside the background error $\boldsymbol{\eta}$ and the observation error $\boldsymbol{\epsilon}$, the deviation of the control parameter from its $a$ priori value $\left(\boldsymbol{\vartheta}_{t}^{c}\right)$ subject to a covariance $\boldsymbol{B}_{c}$ as well,

The spatial support of the control parameter depends on the parameter considered. For an inlet flow control, the parameter is defined on the inlet plane of the computational domain while the coefficient of an error model can be defined over the entire spatial domain. The control parameter can also be stationary or temporally varying, thus requiring, in the latter case, an individual optimisation at each time-step - this can provide, for example, a gradually changing inflow condition capable of better capturing the optimal analysis trajectory.

By applying the adjoint formalism, taking into account the control parameters, the gradient can be evaluated as,

$$
\begin{gathered}
\frac{\partial J}{\partial \boldsymbol{\eta}}=-\boldsymbol{\lambda}_{t_{0}}+\boldsymbol{B}^{-1}\left(\boldsymbol{\mathcal { X }}_{0}^{b}-\boldsymbol{\mathcal { X }}_{0}\right), \\
\frac{\partial J}{\partial \boldsymbol{\vartheta}}=-\boldsymbol{\lambda}_{t_{0}}+\boldsymbol{B}_{c}^{-1}\left(\boldsymbol{\vartheta}-\boldsymbol{\vartheta}_{c}\right)+\left(\partial_{\boldsymbol{\vartheta}} \mathbb{M}\right)^{*} \boldsymbol{\lambda} .
\end{gathered}
$$

The adjoint methodology combined with the 4D-Var approach allows easy addition of control parameters provided the adjoint operator with respect to the 
control parameter $\left(\left(\partial_{\vartheta} \mathbb{M}\right)^{*}\right)$ can be constructed. The $4 \mathrm{D}$-Var algorithm is depicted in figure 1 .

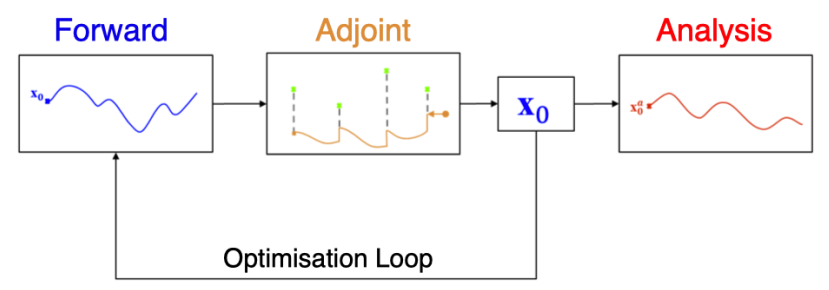

Figure 1: 4D-Var flow chart.

In order to perform data assimilation, the tangent linear and the adjoint version of the flow solver need to be constructed. The practical considerations behind this construction as well as with the optimisation procedure is presented

200

\subsection{Practical considerations}

The construction of the adjoint model, for a dynamical model such as the Navier-Stokes equations, can be performed in two ways: the differentiate-thendiscretise method involves the formulation of a mathematical adjoint which is then discretised on the numerical mesh while the discretise-then-differentiate method constructs the discrete (numerical) adjoint directly on the discretised non-linear model. In literature, the two methods are also referred to as continuous adjoint and discrete adjoint formulations respectively. Given a highly accurate numerical scheme, the discrete adjoint is capable of providing an accurate exact adjoint up to machine accuracy without any assumption (on the boundary conditions, for instance) unlike the continuous adjoint which is in general built from ideal boundary conditions. Thus, the discrete approach is preferred and the adjoint model is constructed using an automatic differentiation (AD) tool TAPENADE [22]. The principles of AD and its application to VDA are briefly 215 explained in the context of flow solvers in Chandramouli [5], Gronskis et al. [20]. Note that for reduced order formulation or ensemble assimilation techniques the 
functional gradient can be directly computed [1, 11] or approximated through finite differences in the subspace spanned by the ensemble members [42, 43, respectively.

The adjoint trajectory in application requires the values of all intermediary variables obtained from the forward trajectory for each optimisation loop. Consider a non-linear dynamical model,

$$
\mathcal{X}_{0} \stackrel{I_{1}}{\longrightarrow} \ldots \stackrel{I_{j}}{\longrightarrow} \mathcal{X}_{j}=I_{j}\left(\mathcal{X}_{j-1}\right) \stackrel{I_{j+1}}{\longrightarrow} \ldots \stackrel{I_{f}}{\longrightarrow} \mathcal{X}_{f}
$$

the corresponding adjoint mode can be computed using,

$$
\boldsymbol{\lambda}_{0} \stackrel{I_{1}^{*}}{\longleftarrow} \ldots \stackrel{I_{j}^{*}}{\longleftarrow} \boldsymbol{\lambda}_{j}=I_{j+1}^{*}\left(\boldsymbol{\mathcal { X }}_{j-1}\right) \boldsymbol{\lambda}_{j+1} \stackrel{I_{j+1}^{*}}{\longleftarrow} \ldots \stackrel{I_{f}^{*}}{\longleftarrow} \boldsymbol{\lambda}_{f} .
$$

Once the tangent linear model and the corresponding adjoint is constructed, the cost function and the gradient can be computed. The optimal solution is then obtained using an iterative optimisation method - the limited storage 

mented in this work.

\subsection{Numerical solver}

The 4D-Var algorithm developed in this work relies on the parallelised numerical solver, Incompact3d, developed by Laizet and Lamballais [25. The solver resolves the full Navier-Stokes equation, i.e. direct numerical simulation (DNS), but its modularity allows for the easy addition of error model functions or turbulence models - this is important in the strong constraint assimilation problem considered here, where the error is introduced in the dynamics. The fortran based solver uses a cartesian mesh and sixth-order compact finite difference schemes for spatial discretisation. The incompressibility constraint is ensured by solving the Poisson equation in spectral space leading to an efficient yet inexpensive solution. The 4D-Var algorithm is enumerated in algorithm 1 and the forward and adjoint trajectories, respecting the modularity of Incompact3d, are depicted in figure 2 .

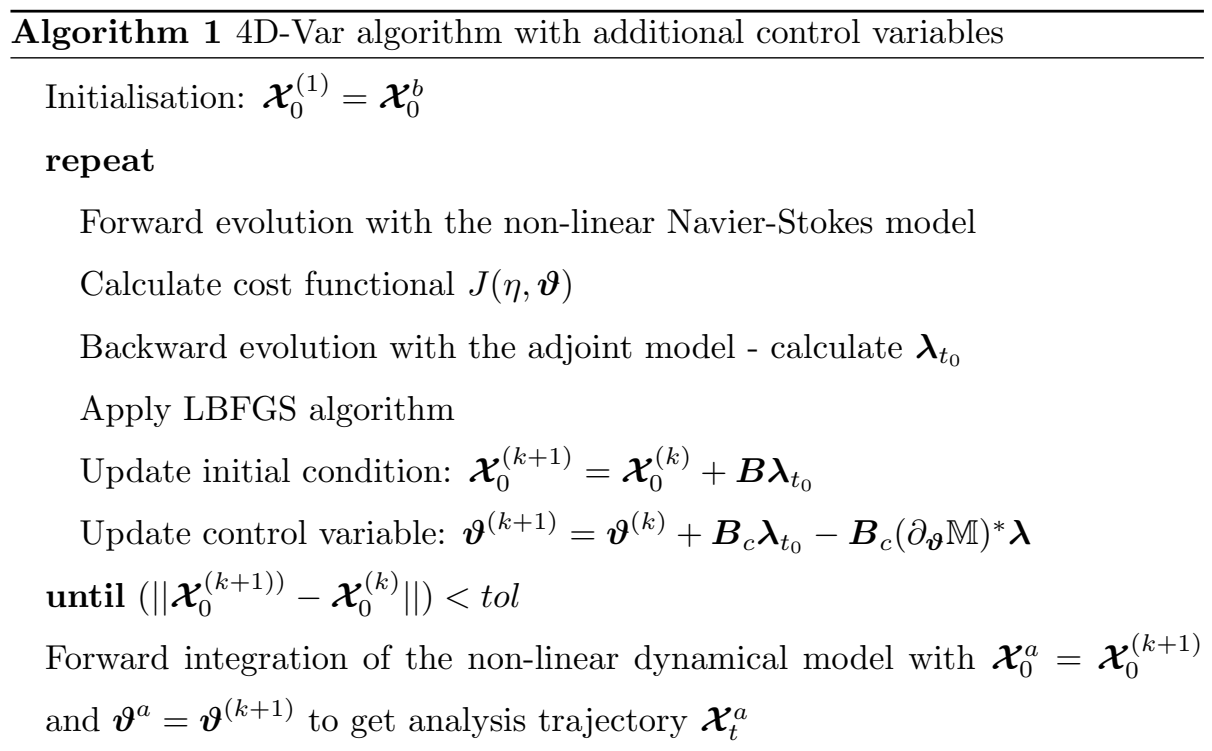



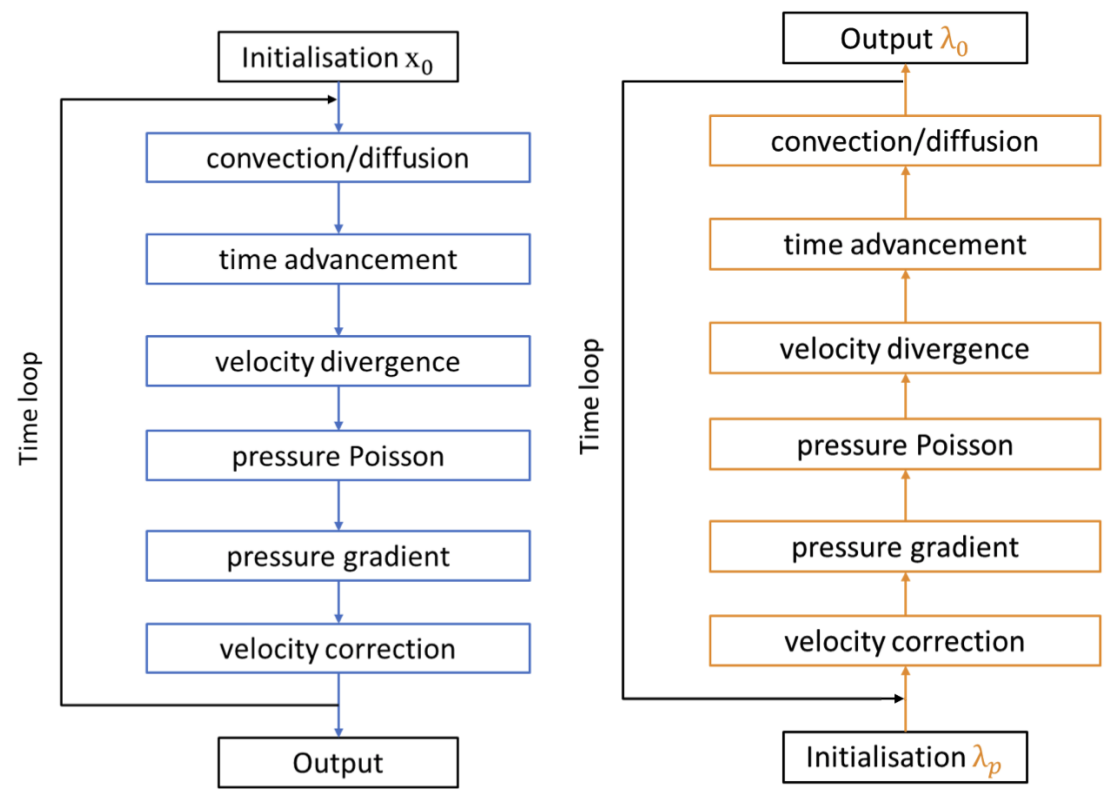

Figure 2: Flow chart for the forward (left) and backward (right) simulation with incompact3d.

\section{Modelling under Location Uncertainty (LU)}

The error modelling considered in the modified dynamics of our 4D-Var strategy is provided by the LU modelling framework of Mémin [29]. The LU model, in its grandest sense, presents a stochastic approach to turbulence modelling based on the decomposition of the velocity into a large-scale smooth component $(\boldsymbol{u})$ and a small-scale highly oscillating random component $\left(\boldsymbol{\sigma}\left(\mathbf{X}_{t}, t\right) \dot{\mathbf{B}}\right)$ representing the instantaneous error of the dynamical system:

$$
\frac{\mathrm{d} \mathbf{X}_{t}}{\mathrm{~d} t}=\boldsymbol{u}\left(\mathbf{X}_{t}, t\right)+\underbrace{\boldsymbol{\sigma}\left(\mathbf{X}_{t}, t\right) \dot{\mathbf{B}}}_{\mathbf{q}_{t}} .
$$

This decomposition of the Lagrangian velocity in terms of a smooth component and a time uncorrelated random error component, leads to a stochastic representation (in the Ito setting) of the rate of change of a scalar quantity transported by the random flow. This is essentially a stochastic representation of the Reynolds transport theorem (RTT) (see Mémin [29, Resseguier et al. [36] for a complete derivation). This stochastic RTT allows us to derive a 
stochastic mass conservation equation and, from the Newton second principle (in a distributional sense), a stochastic Navier-Stokes system of equations for an incompressible fluid [29]

$$
\begin{gathered}
\nabla \cdot \boldsymbol{u}=0 ; \quad \nabla \cdot(\nabla \cdot \mathbf{a})=0 ; \quad \nabla \cdot\left(\mathbf{q}_{t}\right)=0 ; \\
d_{t} \boldsymbol{u}+\left(\left(\boldsymbol{u}^{*} d t+\mathbf{q}_{t}\right) \cdot \nabla\right) \boldsymbol{u}-\frac{1}{2} \nabla \cdot((\mathbf{a} \nabla) \boldsymbol{u}) d t=-\frac{1}{\rho} \nabla p+\nu \Delta\left(\boldsymbol{u} d t+\mathbf{q}_{t}\right),
\end{gathered}
$$

where $d_{t} \boldsymbol{u}$ is the time increment at a given point in space of the velocity, and $\boldsymbol{u}^{*}$ is the modified advection defined as:

$$
\boldsymbol{u}^{*}=\boldsymbol{u}-\frac{1}{2} \boldsymbol{\nabla} \cdot \mathbf{a}
$$

where the variance tensor $\mathbf{a}(\mathbf{x}, t)$ is a $3 \times 3$ symmetric positive definite matrix of finite variation (i.e. they are similar to deterministic functions) directly related to the variance of the error term (i.e. the unresolved velocity component): $\mathbf{a}(\mathbf{x}, t) d t=\mathbb{E}\left(\mathbf{q}_{t}(\mathbf{x}) \mathbf{q}_{t}(\mathbf{x})^{T}\right)$. This variance tensor has the dimensions of kinematic viscosity $\left[\mathrm{m}^{2} \mathrm{~s}^{-1}\right]$ and plays a role similar to the eddy viscosity of classical LES models.

Assuming in addition that the resolved velocity component, $\boldsymbol{u}$, is also of finite variation allows us to safely separate this system in terms of deterministic momentum equations and a stochastic balance:

$$
\begin{gathered}
\partial_{t} \boldsymbol{u}+\boldsymbol{u}^{*} \cdot \nabla \boldsymbol{u}-\frac{1}{2} \sum_{i j} \partial_{x_{i}}\left(\mathrm{a}_{i j} \partial_{x_{j}} \boldsymbol{u}\right)=\frac{1}{\rho} \nabla p+\nu \Delta \boldsymbol{u}, \\
\frac{1}{\rho} \nabla p^{\prime}=-\mathbf{q}_{t} \cdot \nabla \boldsymbol{u}+\nu \Delta\left(\mathbf{q}_{t}\right) .
\end{gathered}
$$

This deterministic system is fully determined by the knowledge of the variance tensor a. The stochastic balance equation (30) enables, with an expression of the diffusion tensor, to get an expression of the turbulent pressure $p^{\prime}$. However, its knowledge is not required in the momentum equation, and this balance equation will not be used further. 
Let us note that relaxing the bounded variation assumption for the resolved component requires to use the full stochastic expression of the momentum equation as in [6, 9.

The set of deterministic conservations equations (eq. (26) and (29)) can be closed by modelling the variance tensor $\mathbf{a}$. Various closure models are presented in Chandramouli et al. 8], of which the stochastic spatial variance (StSp) model was best suited for cylinder wake flow - hence, this is chosen here for modelling a. In this model, the variance tensor is calculated as a local spatial variance based on the resolved velocity field:

$$
\mathbf{a}(\boldsymbol{x}, t)=\frac{1}{|\Gamma|-1} \sum_{x_{i} \in \Gamma(\mathbf{x})}\left(\mathbf{u}\left(x_{i}, t\right)-\mathbf{u}_{m}(x, t)\right)\left(\mathbf{u}\left(x_{i}, t\right)-\mathbf{u}_{m}(x, t)\right)^{T} C_{s p},
$$

where $\mathbf{u}_{m}(x, t)$ stands for the empirical mean on the arbitrarily selected local spatial neighbourhood defined by $\Gamma$. Through dimensional arguments, the coefficient $\mathrm{C}_{s p}$ is defined as [23]:

$$
C_{s p}=\left(\frac{\ell_{\text {res }}}{\ell_{k o l}}\right)^{\frac{5}{3}} \Delta t
$$

270 $\Delta t$ is the simulation time step. In $\$ 5$ this stationary coefficient is assumed to be spatially varying but unknown and is estimated using the 4D-Var algorithm. This coefficient corresponds to the control parameter associated to the model error. An added advantage of using this model in 4D-Var is the namic Smagorinsky sub-grid model which requires filtering and thresholding operations. In order to perform the adjoint of such a model, the corresponding adjoint code formulation needs to be developed accounting for these addition operations which is not straightforward 24 .

The full mathematical derivation of the model can be found in Mémin 29], its successful application to geophysical flows in Resseguier et al. 35, 36, 37. and to industrial/engineering flows in Chandramouli et al. [8], Kadri Harouna and Mémin 23. 


\section{4D assimilation of a wake flow}

285 briefly. This is followed by assimilation results assessing the performance of the algorithm with respect to different parameters: quality of background condition, quality of observations, and background covariance matrices. The assimilation experiments will be conducted in two different situations.

- An idealised case for which a 3D volume of velocity measurements will be

assumed to be available.

- A realistic configuration in which only two orthogonal planes of 3D velocity measurements (2D-3C PIV-like) will be accessible.

The first case will allow us to have an ideal "best" case for the assimilation, to which we will compare the second that corresponds to an experimentally realistic situation.

\subsection{Flow Configuration:}

The experimentation will be conducted on the wake flow over a circular cylinder with reference data. This flow configuration is well-studied for dataassimilation studies and reduced order modelling analyses [17, 41, 20]. To build this reference data, the flow is simulated over a large domain to measure and store velocity fields at regular intervals corresponding to observations. This large domain includes the obstacle whereas the assimilation will be performed on a smaller domain in the wake without any modelling of the obstacle.

305 To include the obstacle geometry in $4 \mathrm{D}$-Var would require the numerical adjoint of the method, such as IBM, used to account for the geometry. However, given no information of the obstacle, to reconstruct the flow field is a unique challenge in its own respect and is what is addressed in this work. All simulations are performed using the parallelised flow solver, Incompact3d.

For obtaining the reference data, the flow over the cylinder is simulated for a Re of 3900 on a domain measuring $20 \mathrm{D} \times 20 \mathrm{D} \times \pi \mathrm{D}$. Due to the restrictive 
cost of DNS, an LES is performed using the LU model (StSp model of Chandramouli et al. 8]) with the domain discretised into $241 \times 241 \times 48$ points with stretching implemented in the lateral direction. In Kolmogorov units $\left(\eta_{k}\right)$, and periodic boundary conditions are implemented in the streamwise, lateral, 
and spanwise directions respectively. The numerical adjoint of these boundary conditions are developed with the $\mathrm{AD}$ tool for the backward trajectory. The choice of free-slip boundary condition is applicable for the assimilation provided that the vortex street does not cross the lateral boundary. The AB3 scheme and its adjoint are used for the forward and backward trajectories respectively.

The non-stretched mesh together with the imprecise boundary conditions yields inherent discrepancy between the reference model and the assimilation error model. The assimilation is carried out on this domain using the StSp model (eq. (31) for the variance tensor a) for which a constant initial coefficent is fixed based on the scaling of eq. (32). It is important to note that only the three-component velocity fields will be observed in the assimilation window. All the other quantities required for the restart procedure of Incompact3d at the beginning of the assimilation window (pressure field, convection and diffusion terms of previous time-steps, etc) are assumed to be completely unknown and hence set to zero. This contributes as well to an additional strong discrepancy between the reference and assimilation models. The spatial parameters for the reference and assimilation domain are tabulated in table 1 .

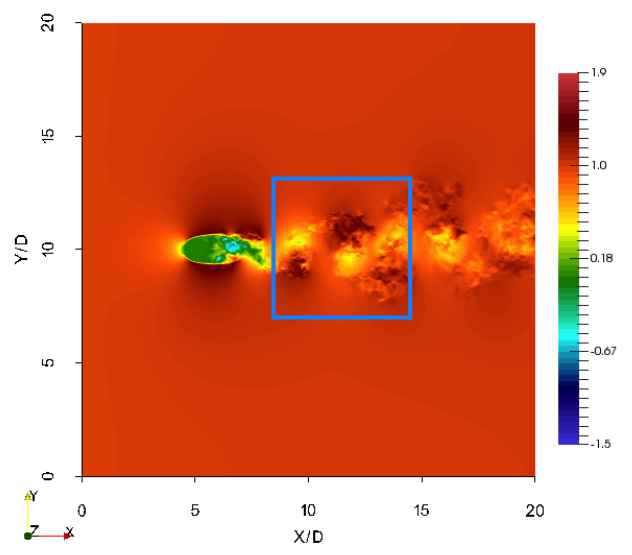

Figure 3: Streamwise velocity snapshot in the reference domain with the cylinder at a (x,y) of $(5 \mathrm{D}, 10 \mathrm{D})$. The sub-domain in blue corresponds to the assimilation domain; Observation data are extracted in this domain. 
Table 1: Configuration parameters for the reference and assimilation runs.

\begin{tabular}{cccccc}
\hline & $\mathrm{Re}$ & $\mathrm{n}_{x} \times \mathrm{n}_{y} \times \mathrm{n}_{z}$ & $\mathrm{l}_{x} / \mathrm{D} \times \mathrm{l}_{y} / \mathrm{D} \times \mathrm{l}_{z} / \mathrm{D}$ & $\mathrm{U} \Delta t / \mathrm{D}$ & Duration \\
\hline Reference data constitution & 3900 & $241 \times 241^{s} \times 48$ & $20 \times 20 \times 3.14$ & 0.003 & $30100 \Delta t$ \\
Data extraction & 3900 & $165^{i} \times 165^{i} \times 48$ & $6 \times 6 \times 3.14$ & 0.003 & $100 \Delta t$ \\
4D-Var & 3900 & $165 \times 165 \times 48$ & $6 \times 6 \times 3.14$ & 0.003 & $100 \Delta t$ \\
\hline \multicolumn{5}{c}{ s - Stretched; i - Interpolated }
\end{tabular}

360 via the poisson solver. However, such a propagation accepts a significantly wide 
range of acceptable solutions which may not be close to the true state of the system. In this work, we opt rather for the fast snapshot optimisation (SO) algorithm proposed by Chandramouli et al. [7] to reconstruct the unknown 3D volume given the information on the two cross-planes $\left(\mathrm{Obs}_{\text {rec }}\right)$. The SO method exploits the homogeneity along the spanwise direction for cylinder wake in order to identify from a long sequence of $2 \mathrm{D}$ observations the optimal match using a gradient descent methodology. Such an algorithm manages to reconstruct major turbulent structures in the flow with a low computational cost. The advantage of such a reconstruction lies in the additional information that is provided to the assimilation algorithm - namely the presence of temporal and spanwise spatial homogeneity. An alternative weighted gaussian interpolation methodology is also used to reconstruct the 3D domain $\left(O b s_{\text {inter }}\right)$ given $2 \mathrm{D}$ planar fields to facilitate a comparison with the SO reconstructed observations (see appendix for a description of the interpolation method used). For both of these observations, the confidence on the data is reflected on $\boldsymbol{R}_{P I V}^{-1}$ with reduced values at all points except on the two cross-planes of true data. A 2D $(X Y)$ view of the three types of observations at $z=1.31 D$ are shown in figure of observations are shown in figure 5 - to reiterate, $\boldsymbol{R}_{3 D}^{-1}$, corresponding to the 'ideal' case, retains maximum confidence on the data within the assimilation domain with reduced confidence on the boundaries while $\boldsymbol{R}_{P I V}^{-1}$, corresponding to the 'realistic' case, reflects high confidence only for data on the two planes on which the three components of velocity are extracted (the precise expression for $\boldsymbol{R}_{3 D}^{-1}$ and $\boldsymbol{R}_{P I V}^{-1}$ are provided in the appendix). The offset for visualisation from the mid-plane at $z=1.57 D$ is needed to emphasise the difference between the three kinds of observations which are identical in the spanwise mid-plane. Henceforth all velocity field visualisation are done on the $X Y$ plane at $z=1.31 D$ unless mentioned otherwise.

Discontinuities can be seen in the velocity fields with the interpolation algorithm that are non-physical. This artefact is strengthened in the outlet velocity fields shown in figure 6 for the three observations. The interpolation downstream 

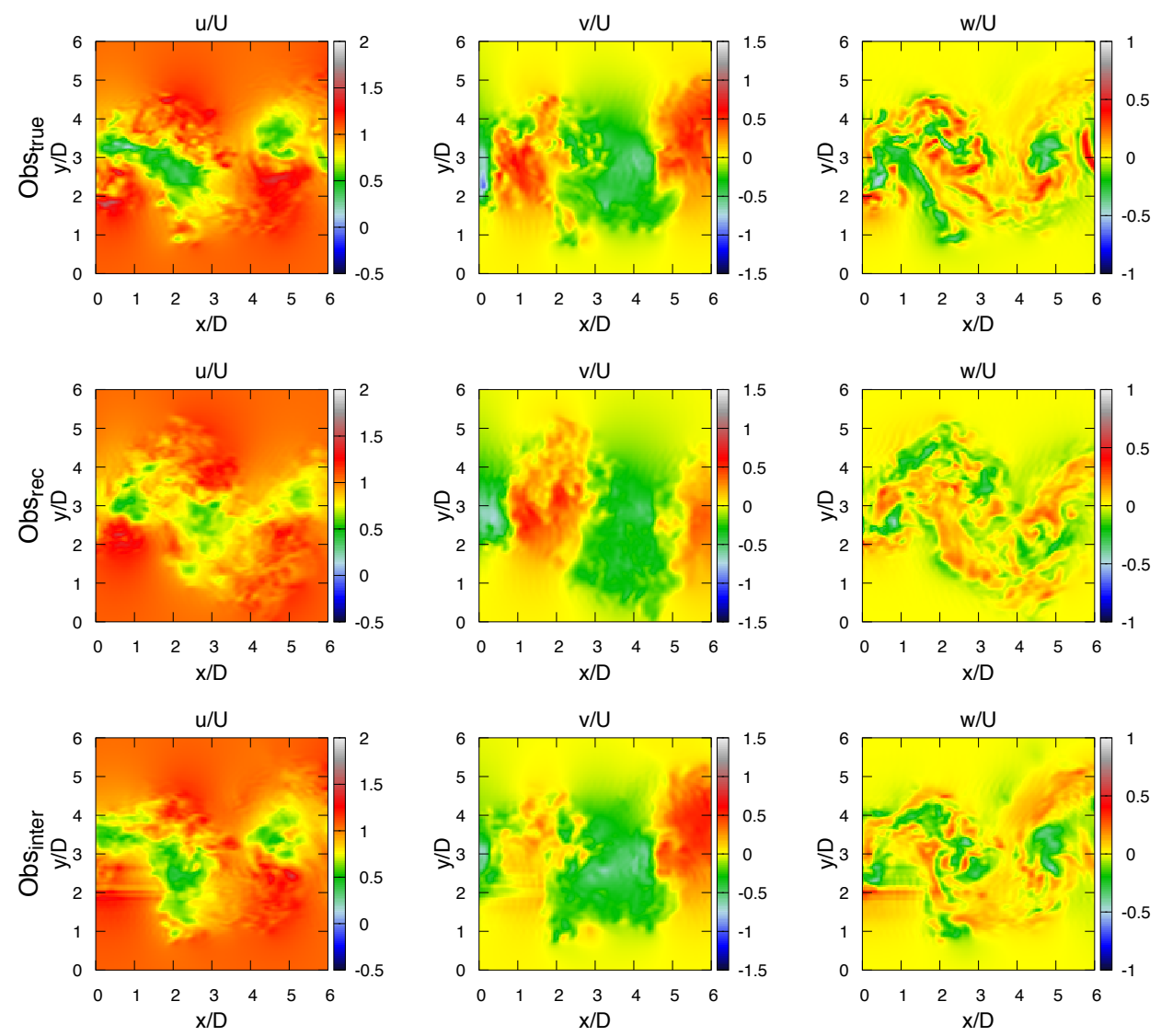

Figure 4: Observation velocity snapshots at the beginning of the assimilation window $\left(t_{1}^{\prime}\right)$ in the plane $z=1.31 \mathrm{D}$. From left to right: streamwise, lateral, and spanwise fields. From top to bottom: $O b s_{\text {true }}, O b s_{r e c}$, and $O b s_{\text {inter }}$

from the inlet simply mirrors the observation plane leading to unphysical observations. The $\mathrm{SO}$ algorithm is able to roughly reconstruct turbulent structures that are representative of the flow. The effect of the quality of observations on the assimilation algorithm is analysed in 4.4 .2

\subsection{Background data}

An accurate background condition provides a good starting point for the assimilation algorithm leading to faster convergence while an inaccurate condition, which is too far from the optima, can restrict and destabilise the algorithm. For 

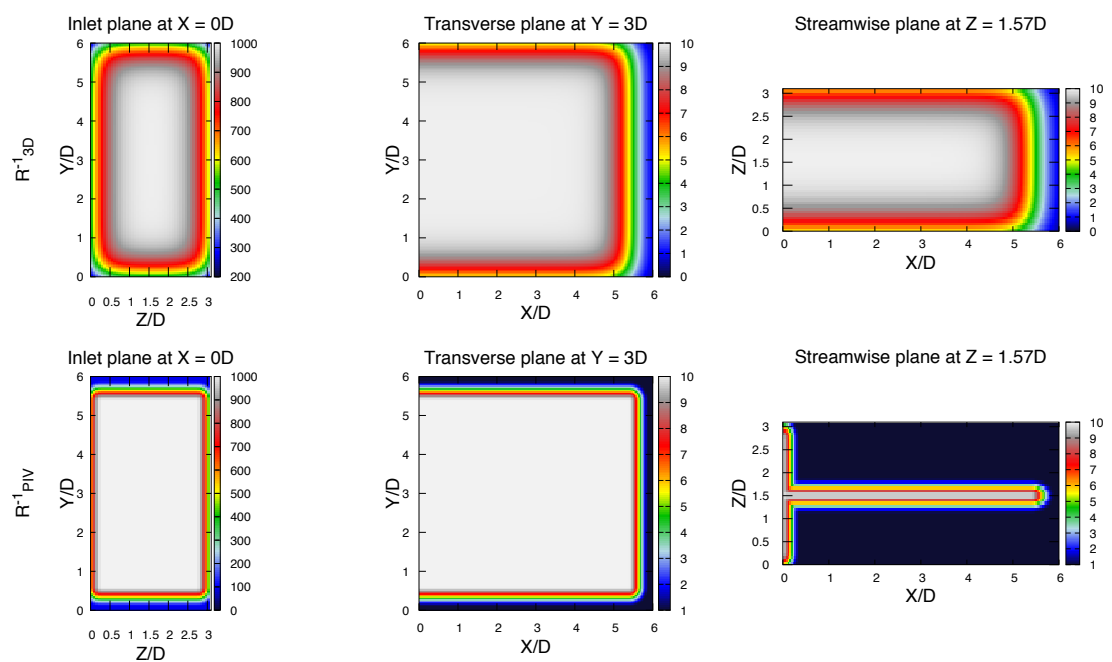

Figure 5: Confidence on the observed velocity fields denoted by $\boldsymbol{R}^{-1}$. From left to right: inlet plane at $x=0 D$, transverse plane at $y=3 D$, and streamwise plane at $z=1.57 D$. From top to bottom: $\boldsymbol{R}_{3 D}^{-1}$, and $\boldsymbol{R}_{P I V}^{-1}$

initial tests with volumetric observations, the background condition is obtained by biasing the true velocity maps by a sinusoidal wave. This 'incorrect' or biased background ( $\left.B g_{\text {bias }}\right)$ condition provides an opportunity to test algorithm stability and accuracy in the validation study to follow.

Experimentally, it is hardly feasible, and only at high computational cost, to have 3D observations from which a background condition can be obtained. Such a 3D initial background, which is required for 4D-Var, can be obtained through 3D tomographic PIV techniques [12] or through numerical simulation, where the flow is simulated from a precise, temporally highly resolved, inlet condition until the entire domain is filled. Both techniques are computationally very costly. An alternate solution, drawing inspiration from the observation reconstruction, is to use the SO method to reconstruct the volume with 2D planar data. Such a background condition $\left(B g_{r e c}\right)$ can be obtained in a very fast way and constitute 435 a good representation of the flow condition. The three components of the two background conditions are shown in figure 7. The dependancy of the 4D-Var algorithm on the quality of the background condition is analysed in 4.4 .1 . 

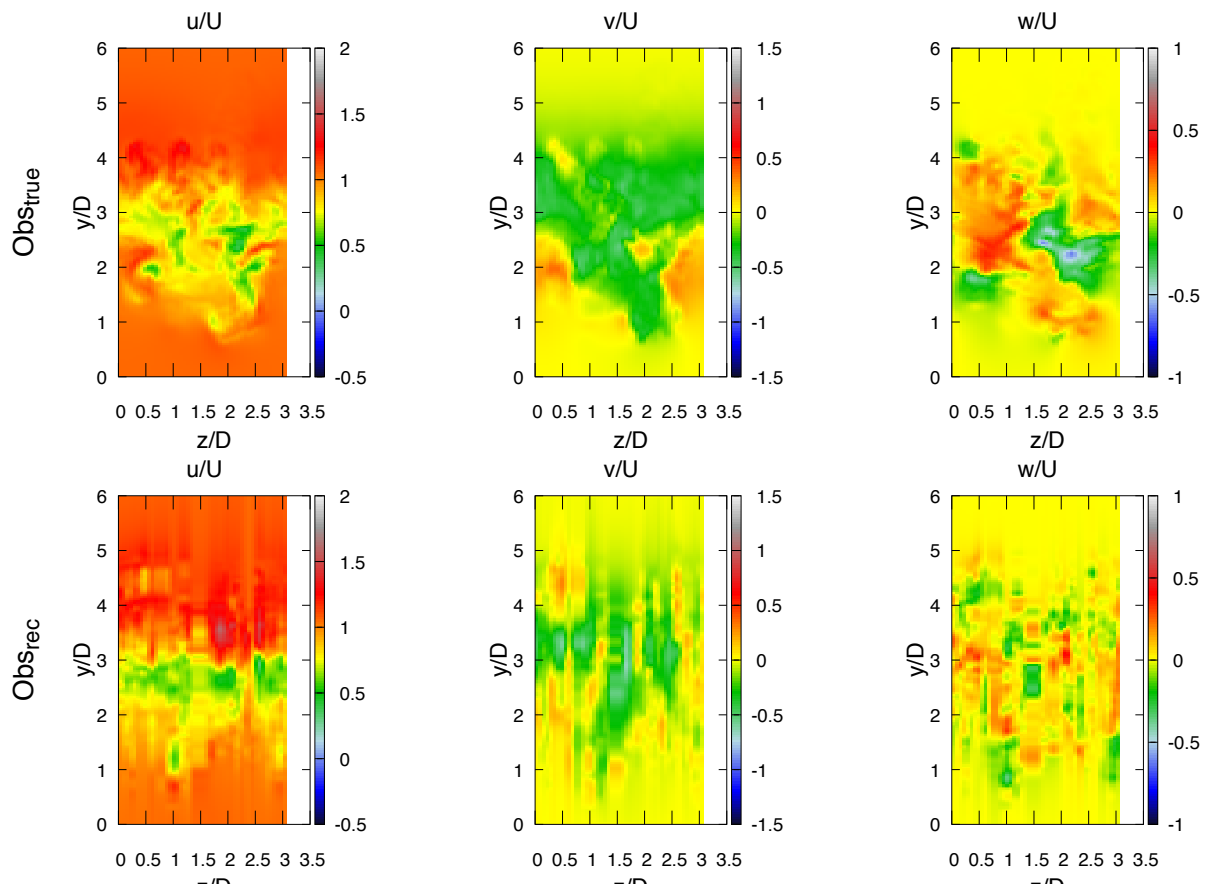

$\mathrm{v} / \mathrm{U}$
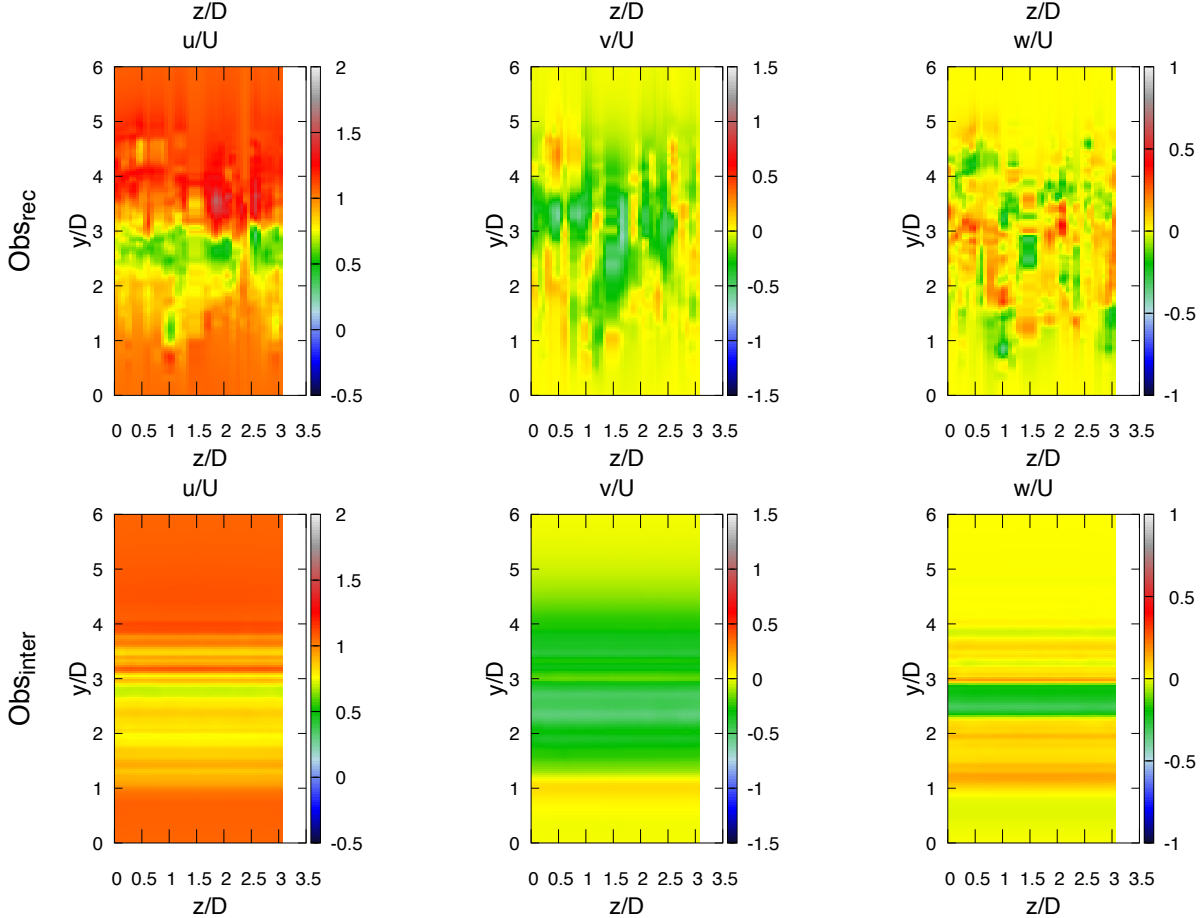

Figure 6: Observation velocity snapshots at the beginning of the assimilation window $\left(t_{1}^{\prime}\right)$ in the plane $x=6 \mathrm{D}$ (at the outlet). From left to right: streamwise, lateral, and spanwise fields. From top to bottom: Obstrue, $O b s_{r e c}$, Obsinter.

The definition of the inverse of the background covariance matrix $\boldsymbol{B}^{-1}$ and the associated implication on the 4D-Var algorithm is dealt with in section 4.4 .3 $440 \quad$ For results presented in 4.4 .1 and 4.4 .2 this matrix is set to be identity - i.e. each point in space is independent of other points in space and a change at one point has no direct effect on the neighbouring points. We present now the 

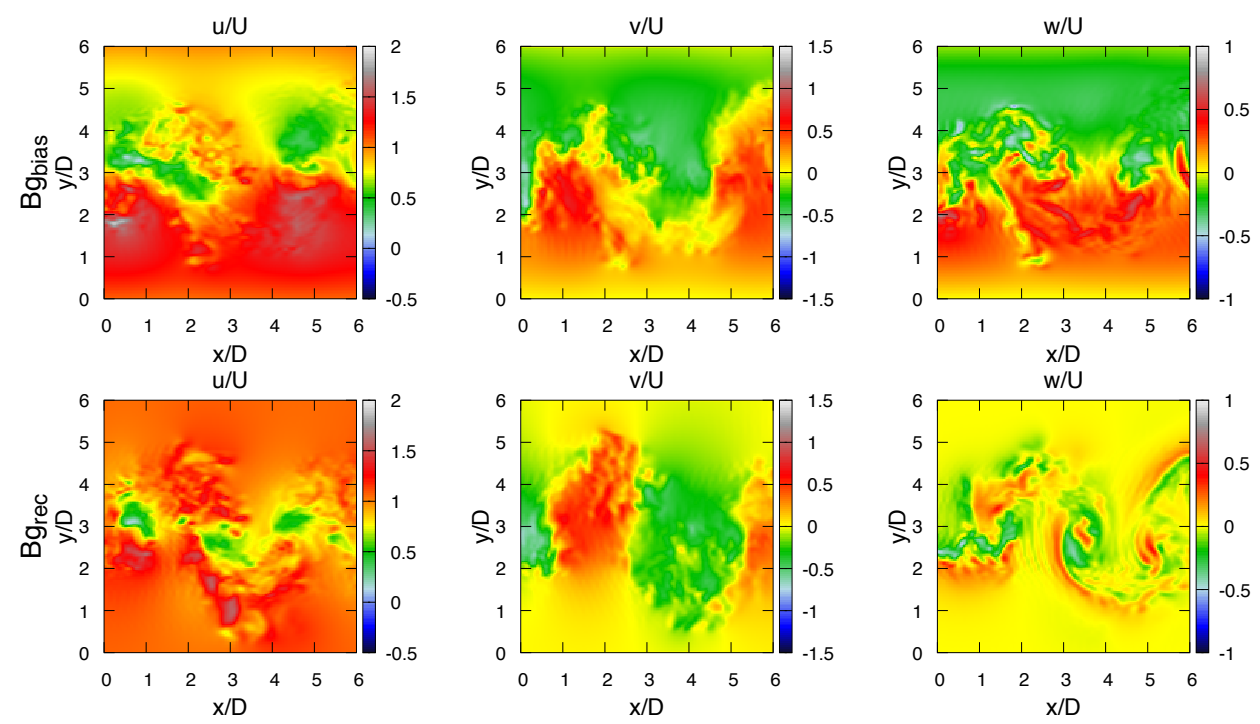

Figure 7: Background velocity snapshots at the beginning of the assimilation window $\left(t_{1}^{\prime}\right)$ in the plane $z=1.31 \mathrm{D}$. From left to right: streamwise, lateral, and spanwise fields. From top to bottom: $B g_{\text {bias }}$, and $B g_{r e c}$

algorithm results via an analysis of three essential parameters for 4D-Var: the background condition, the observation data-set, and the background covariance matrix.

\subsection{D-Var results}

\subsubsection{Analysis 1 - Background condition}

The first parameter analysed is the background condition and the effect of its quality on the assimilation results. This section aims to answer the following questions:

- How important is the quality of the background condition?

- What is its effect in terms of accuracy and cost for $4 \mathrm{D}$-Var?

- Is algorithm stability correlated with the background condition?

We perform two simulations with identical parameters using Obstrue for both while varying the background condition. For case (a), we use the biased 
Table 2: Parameters for the different assimilation cases.

\begin{tabular}{ccccc}
\hline Case & Observations & Background & Background covariance & Coefficient optimisation \\
\hline (a) & Obs $s_{\text {true }}$ & $B g_{\text {bias }}$ & Identity & No \\
(b) & Obs $s_{\text {true }}$ & $B g_{\text {rec }}$ & Identity & No \\
(c) & Obs $s_{\text {inter }}$ & $B g_{\text {rec }}$ & Identity & No \\
(d) & $O b s_{\text {rec }}$ & $B g_{\text {rec }}$ & Identity & No \\
(e) & Obs $s_{\text {true }}$ & $B g_{\text {rec }}$ & Fully-defined & No \\
(f) & Obs rec & $B g_{\text {rec }}$ & Fully-defined & Yes \\
\hline
\end{tabular}

background while for case (b) we use the reconstructed background using the SO methodology. The parameters of all cases studied in this article are presented concisely in table 2. Neither background condition is a perfect representation of the initial flow state but it can be argued that the deviations introduced by the bias are non-physical while the reconstructed rough background only introduces a phase-change - i.e. a representation of the flow at a different time with minor discontinuities in the spanwise directions. Case (a) is a test for the algorithm in terms of stability and capability to recover the reference state given an incorrect initial condition. Case (b) aims to explore the importance of the quality of the background condition and its effect on the accuracy and cost of the 4D-Var algorithm.

The optimisation characteristics, namely the cost function $\left(J\left(\mathcal{X}_{0}\right)\right)$ and its gradient $\left(\left\|\nabla J\left(\mathcal{X}_{0}\right)\right\|\right)$, are plotted as a function of the inner loop iterations in figure 8 As can be expected, a direct correlation is observed between the quality of the background and the iterations required to converge to a solution - case (b) requires only half of the iterations of case (a) to converge. The final cost of the analysis trajectory (analysis refers to the final estimate of the velocity field obtained from the assimilation) are similar for both cases. For case (a), the mismatch between the observations and the background manifests as peaks in the cost functional compared to the smooth decline for case (b). These peaks occur 
due to very strong gradients, which alter the gradient descent procedure. These gradients can be associated to the unphysical background condition provided to the procedure which does not respect the dynamical model.
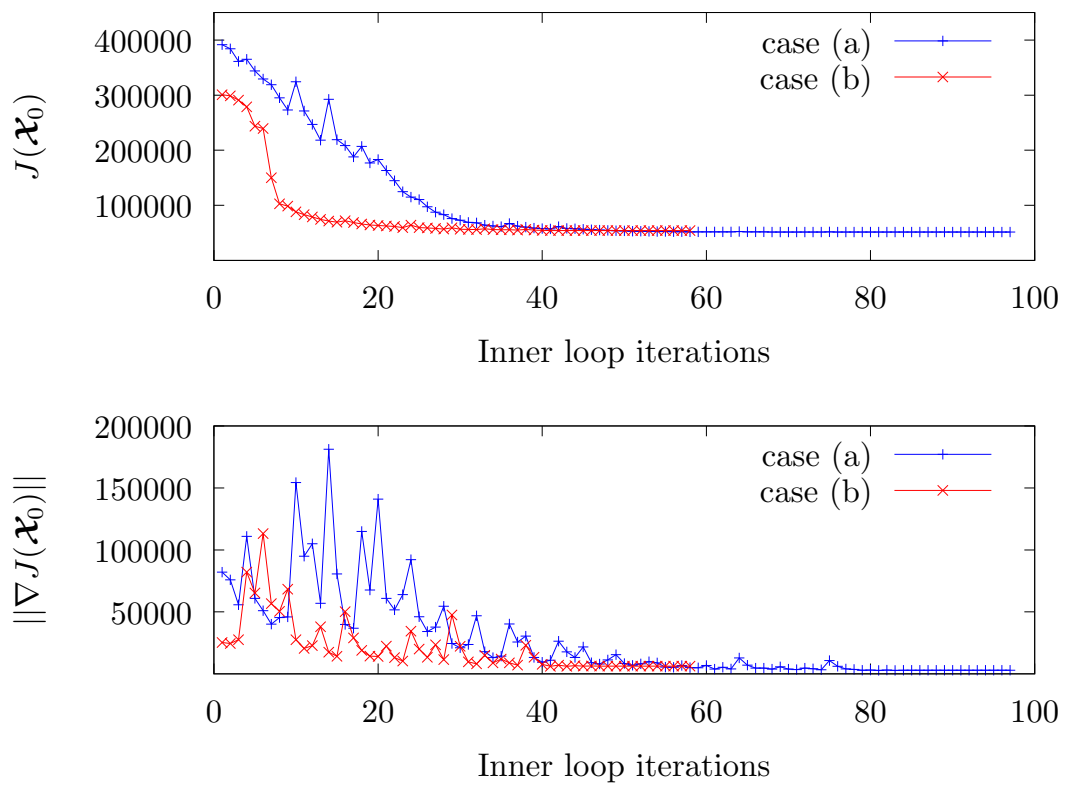

Figure 8: Evolution of the cost function $J\left(\mathcal{X}_{0}\right)$ and its gradient $\left\|\nabla J\left(\mathcal{X}_{0}\right)\right\|$ for the assimilation as a function of inner loop iterations for cases (a) and (b).

The analysis velocity field maps for the two cases $\left(A n_{\text {case }(a)}\right.$, and $\left.A n_{\text {case (b) }}\right)$, are shown in figure 9 along with the corresponding background maps and the reference state. There is a marked improvement from the background to the analysis contour maps especially for case (b) where we observe a near-perfect match with the reference. The analysis maps suggests that an incorrect but physically meaningful representation of the flow enables to improve the accuracy of the assimilation results. The algorithm, while remaining globally stable (i.e. the sharp peaks due to high gradients do not alter the global convergence) for the biased background, does not manage to identify an accurate initial condition. However, at the end of the assimilation window, we obtain velocity maps for both cases that match well with the reference (see figure 10). This result highlights 

incorrect data-sets.

\subsubsection{Analysis 2 - Observation data-set}

$3 \mathrm{D}$, accurate measurements of variables of interest in the assimilation domain are difficult to obtain with existing methodologies. While simulations can provide these quantities, their accuracy will be dependant on the boundary condition errors and other numerical errors. 3D flow measurement techniques, such as tomo-PIV, are computationally expensive techniques and are still restricted in their application to small domain or sparse data. However, to obtain 2D3C data on cross-planes, quick and efficient established stereoscopic PIV techniques are

Identical flow and optimisation parameters are provided to both cases with the $B g_{r e c}$ background condition. In both cases, the $3 \mathrm{D}$ reference has not been provided. Both the observations and the background are only an expectation of the $3 \mathrm{D}$ flow field as is the case with assimilation of experimental observations.

The optimisation characteristics in figure 11 show similar convergence for both cases with a few additional iterations for case (d) and a final cost that is marginally higher for case (c) as compared to case (d). For case (c), we note 

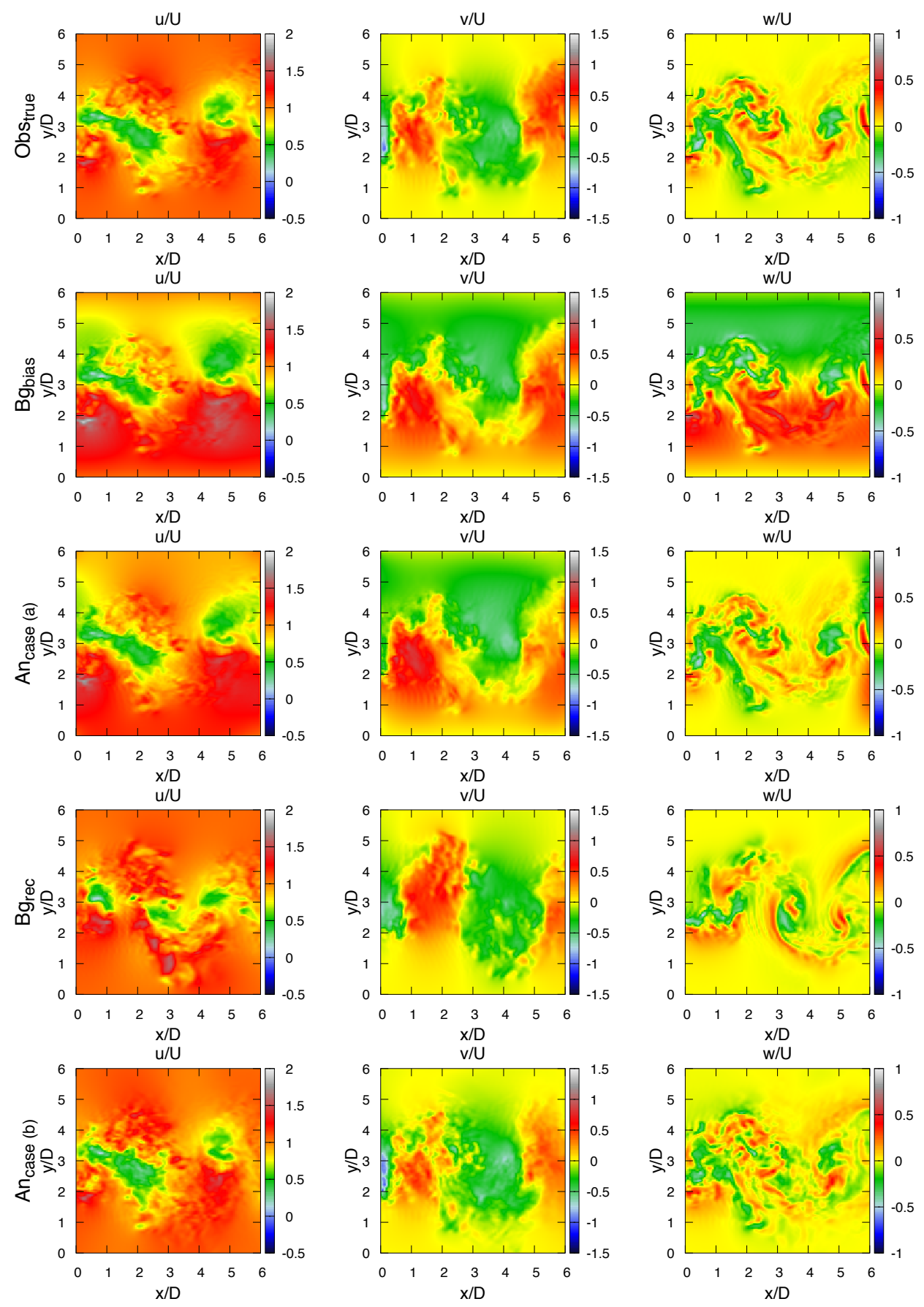

Figure 9: Velocity snapshots at the beginning of the assimilation window $\left(t_{1}^{\prime}\right)$ in the plane $z$ $=1.31 \mathrm{D}$. From left to right: streamwise, lateral, and spanwise fields. From top to bottom: Obstrue, $B g_{\text {bias }}, A n_{\text {case (a) }}, B g_{\text {rec }}$ and $A n_{\text {case (b) }}$ 

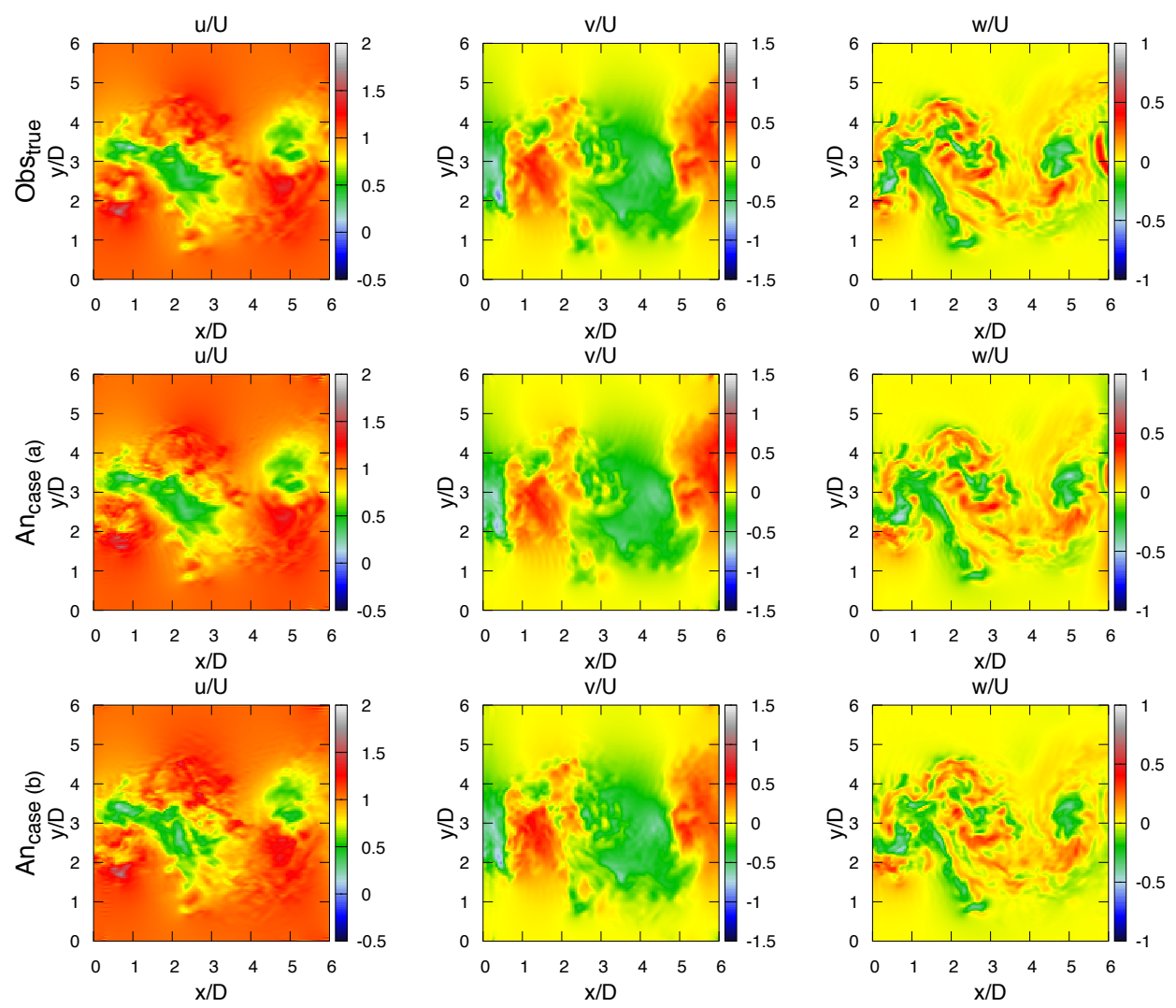

Figure 10: Velocity snapshots at the end of the assimilation window $\left(t_{1}^{\prime}\right)$ in the plane $z=$ 1.31D. From left to right: streamwise, lateral, and spanwise fields. From top to bottom: Obstrue, $A n_{\text {case (a) }}$, and An case (b)

that a high gradient peak perturbates the gradient descent which is likely due to unphysical observations associated to the interpolation procedure. These characteristics suggests that simple mathematical techniques such as interpolation methods provide satisfactory observations for assimilation. We also observe that the analysis velocity maps obtained with both cases (see figure 12 are quite similar - the analysis is a compromise between the background and the observations, constrained by the governing equations. The forward evolution in time of the full nonlinear dynamics from the analysis initial condition, however, introduces instabilities for case (c) leading to a 
numerical explosion unlike case (d) which remains stable. These instability issues, due again probably to unphysical forcing, prevent thus the practical use of interpolated data. The analysis velocity maps for case (d) at the end of the assimilation window is shown in figure 13 .
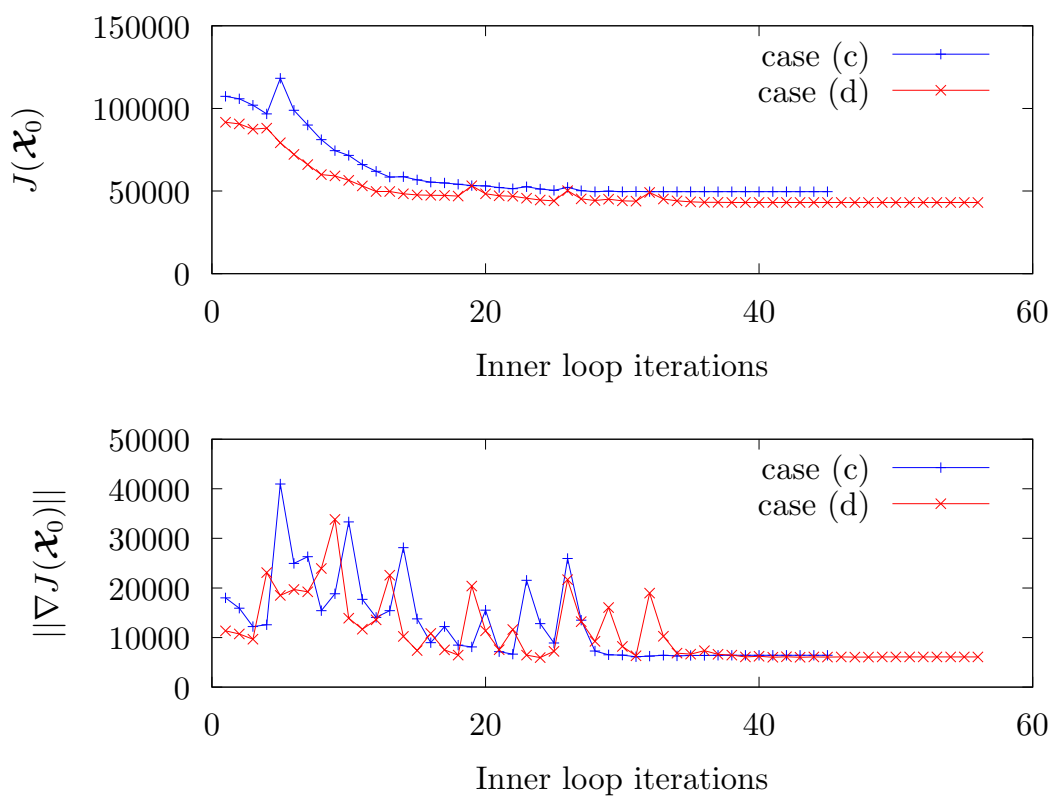

Figure 11: Evolution of the cost function $J\left(\mathcal{X}_{0}\right)$ and its gradient $\left\|\nabla J\left(\mathcal{X}_{0}\right)\right\|$ for the assimilation as a function of inner loop iterations for cases (c) and (d).

The root-mean-square-error (RMSE) evolution along time between the background, observation, and analysis trajectories with the true observations are plotted in figure 14 for the three velocity components for case (d). The RMSE for the analysis (in red) has the lowest error with significant improvement over the background. The analysis is the best estimate for the spanwise component as the observations, which are constructed using the SO algorithm, do not ensure a divergence free flow. The $4 \mathrm{D}$-Var analysis is subject to the divergence free criterion by the solver and thus predicts an improved trajectory for the spanwise velocity component. 

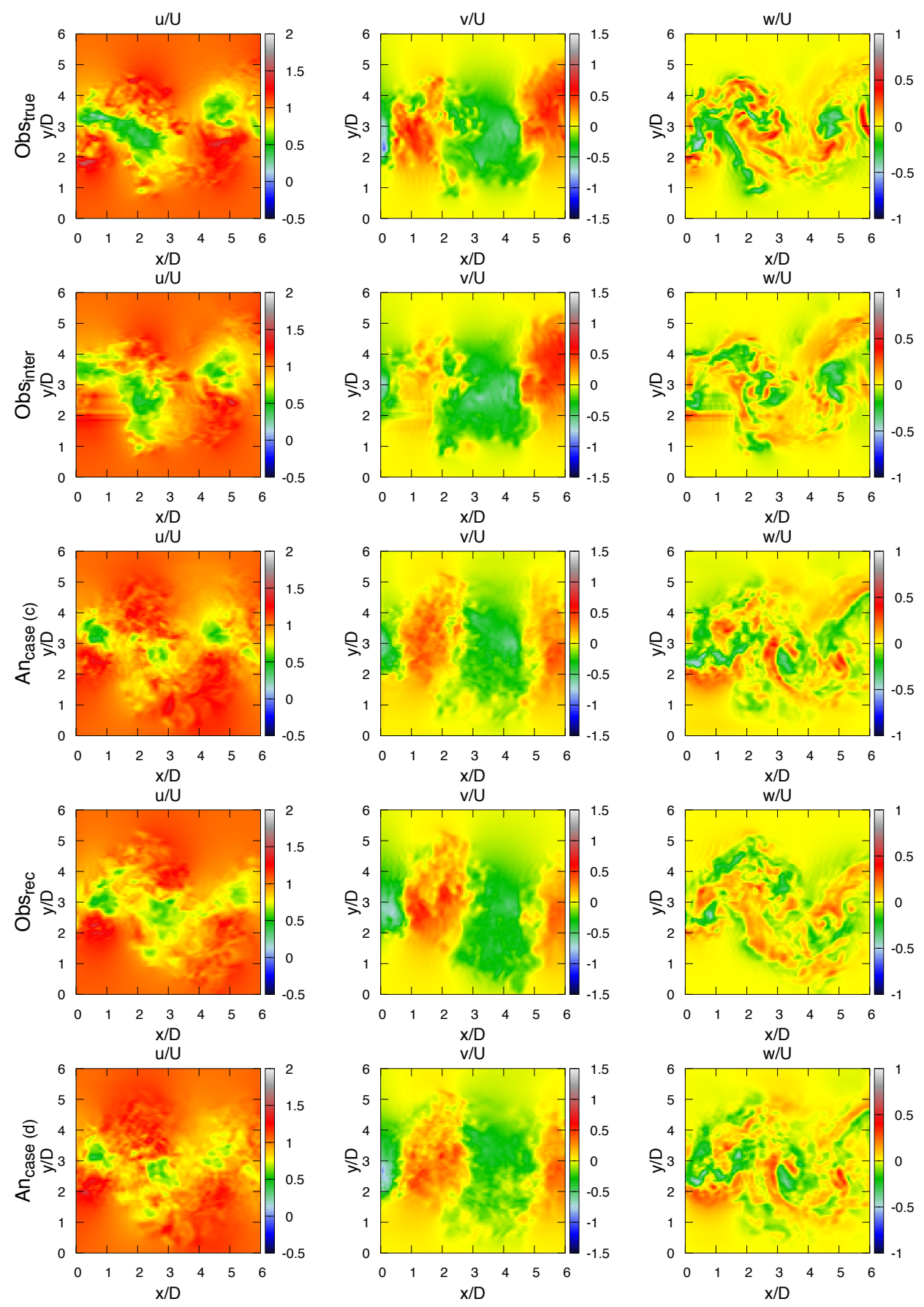

Figure 12: Velocity snapshots at the beginning of the assimilation window $\left(t_{1}^{\prime}\right)$ in the plane $z=1.31 \mathrm{D}$. From left to right: streamwise, lateral, and spanwise fields. From top to bottom: Obstrue, $O b s_{\text {inter }}, A n_{\text {case (c) }}, O b s_{\text {rec }}$ and $A n_{\text {case (d) }}$ 

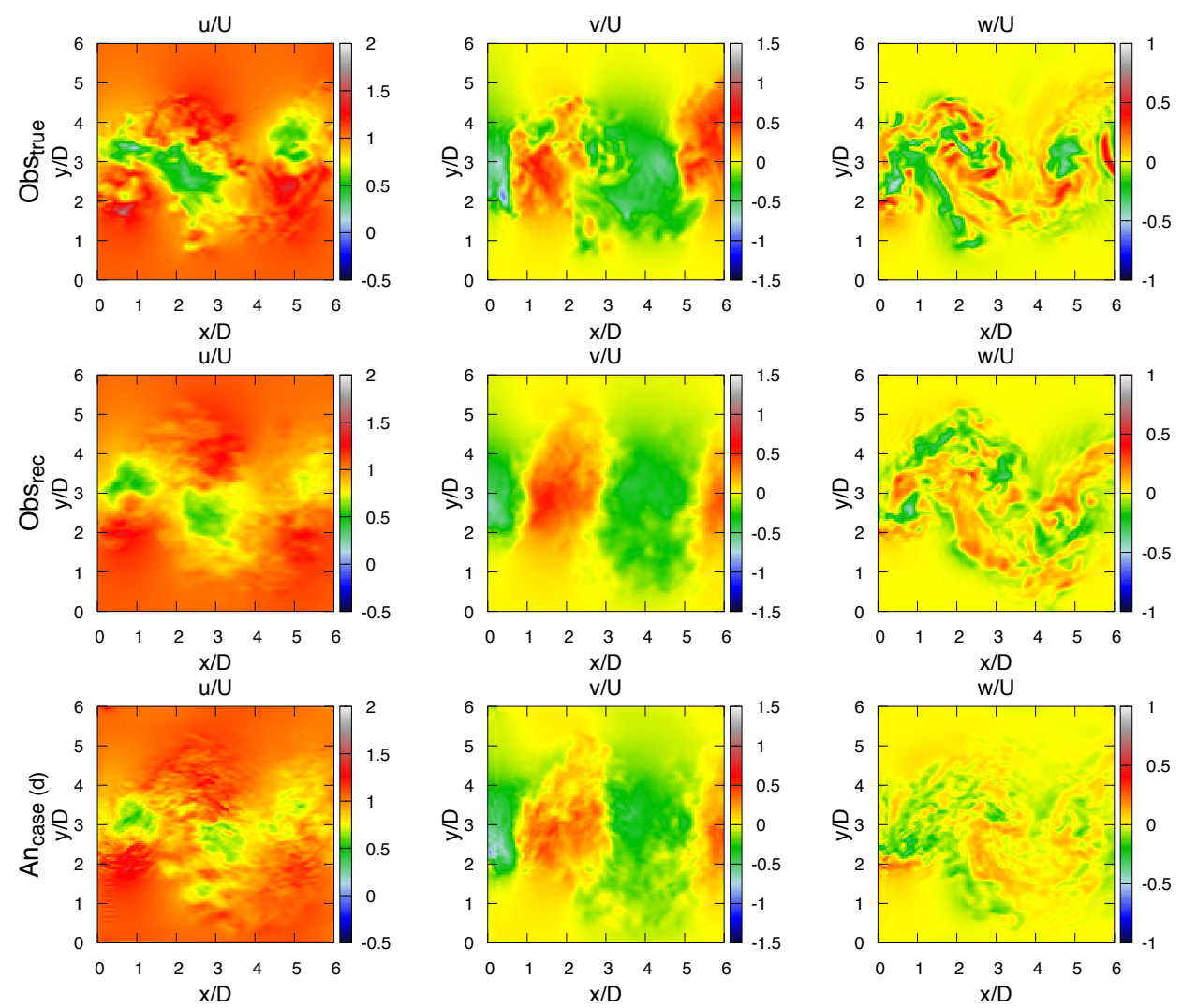

Figure 13: Velocity snapshots at the end of the assimilation window $\left(t_{2}^{\prime}\right)$ in the plane $z=$ 1.31D. From left to right: streamwise, lateral, and spanwise fields. From top to bottom: Obstrue, $O b s_{r e c}$, and $A n_{\text {case }}(c)$

\subsubsection{Analysis 3-Background covariance}

The use of an accurate, well-defined background covariance matrix $\boldsymbol{B}$ is expected to provide significant improvements to the efficiency of the algorithm with faster convergence. However, the construction of the $\boldsymbol{B}^{-1}$ matrix is usually cumbersome, requiring extensive computations and/or memory due to its considerable size. In this section, we use an innovative method, assisted by the $\mathrm{SO}$ algorithm, for estimating this matrix using multiple reconstructions.

Consider the fully synthetic case with 3D observations using SO reconstructed background initial condition. The data-collection step is run for a 

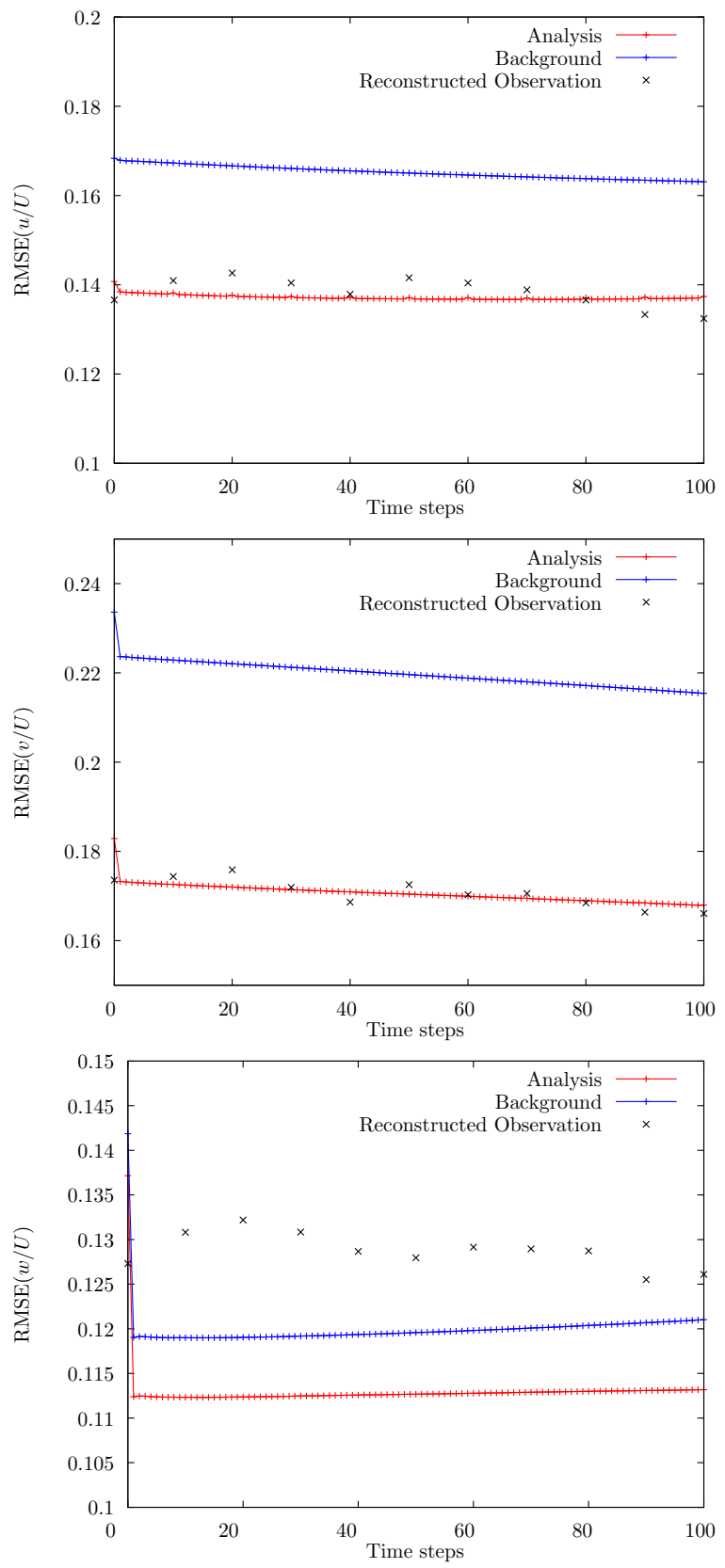

Figure 14: Evolution of the RMSE along time of the three velocity components between the true observations and the assimilation components, namely background, observation, and analysis for case (d). 
long period producing 4000 snapshots with each snapshot spaced 50 time steps apart. The 2D3C cross-plane data is extracted from the 4000 snapshots for use with the SO algorithm. Instead of using all the 4000 snapshots for one single reconstruction, we use a sliding window for the number of OP snapshots used to create multiple reconstructions i.e. we use the first 400 OP snapshots for reconstruction \#1, 200-600 for \#2, 400-800 for \#3, and so on giving us 19 reconstructed $3 \mathrm{D}$ velocity fields at one instant of time $t_{1}^{\prime}$. Removing the mean and decomposing the set of reconstructed fields using singular value decomposition (SVD) gives an estimate for the background covariance matrix through the velocity fluctuations $\left(\boldsymbol{u}^{\prime}\right)$ as,

$$
\boldsymbol{u}^{\prime}=U S V^{T}
$$

where $U$ contains the left singular vectors as columns, $V^{T}$ contains the right singular vectors as rows, and $S$ is a diagonal matrix composed of the singular values in decreasing order of magnitude. The background covariance matrix and its inverse can then be defined as,

$$
\begin{gathered}
\boldsymbol{B}=\boldsymbol{u}^{\prime} \boldsymbol{u}^{\prime T}=\left(U S V^{T}\right)\left(U S V^{T}\right)^{T}=U S^{2} U^{T}, \\
\boldsymbol{B}^{-1}=\left(U S^{2} U^{T}\right)^{-1}=U S^{-2} U^{T}, \\
\boldsymbol{B}^{-\frac{1}{2}}=U S^{-1} U^{T} .
\end{gathered}
$$

With the computed $\boldsymbol{B}^{-\frac{1}{2}}$, the components of the cost function and its gradient with respect to the background can be modified as,

$$
\begin{gathered}
J(\boldsymbol{\eta})=\frac{1}{2}\left\|\mathcal{X}_{0}^{b}-\mathcal{X}_{0}\right\|_{B^{-1}}^{2}=\frac{1}{2}\left(\left\|\mathcal{X}_{0}^{b}-\mathcal{X}_{0}\right\| B^{-\frac{1}{2}}\right)\left(B^{-\frac{1}{2}}{ }^{T}\left\|\mathcal{X}_{0}^{b}-\mathcal{X}_{0}\right\|^{T}\right), \\
\frac{\partial J}{\partial \boldsymbol{\eta}}=\left(\delta \mathcal{X}_{0} \boldsymbol{B}^{-\frac{1}{2}}\right)\left(\left(\boldsymbol{B}^{-\frac{1}{2}}\right)^{T}\right) .
\end{gathered}
$$

Such an evaluation takes into account the effect that a change in velocity at one point has on other points in a neighbourhood through the inverse of the 
$\boldsymbol{B}$ matrix. This should result in faster convergence due to a distributed optimisation procedure where convergence at each mesh-point assists in obtaining a better estimate of the field at other points with respect to the background condition.

This is studied by performing an assimilation run (case (e)) with identical parameters to case (b). The difference between the two cases lies in the definition of the $\boldsymbol{B}^{-1}$ matrix which was set to identity in case (b) but is estimated in case (e). The cost functional and its gradient evolution for the two cases are shown in figure 15 A significantly faster convergence is obtained with case (e) (26 inner loop iterations) requiring only half the iterations of case (b) (58 inner loop iterations). The final cost is marginally higher in this case due to the increased weighting of the background error caused by the fully defined background covariance matrix. The gradient of the cost function for the initial set of iterations are identical for both cases but as the optimal trajectory deviates further from the background, the covariance matrix plays a role and thus, the two gradient curves deviate from each other. These characteristics suggest that a good estimate of the background covariance matrix provides a significant computational cost reduction by improving the efficiency of the optimisation algorithm.

The optimal analysis provided by the fully-resolved $\boldsymbol{B}$ matrix case is shown in figure 16 at time $t_{1}^{\prime}$. The corresponding analysis for case (b) is reproduced here for easy comparison. We can see that both analysis trajectories are comparable and match well with the reference. The RMSE evolution, shown in figure 17, highlights the slight differences between the two cases. The assimilation algorithm improves on the background condition with either covariance matrix, however, the analysis from case (b) is of lower error than case (e). This discrepancy, despite better information being provided through the background covariance matrix, could be due to a lack of detailed information on the observations e.g. lack of a well-defined observation covariance matrix. In the authors opinion, the significant reduction in computation cost obtained with a well-defined covariance off-sets the loss of accuracy in the analysis. In addition, 

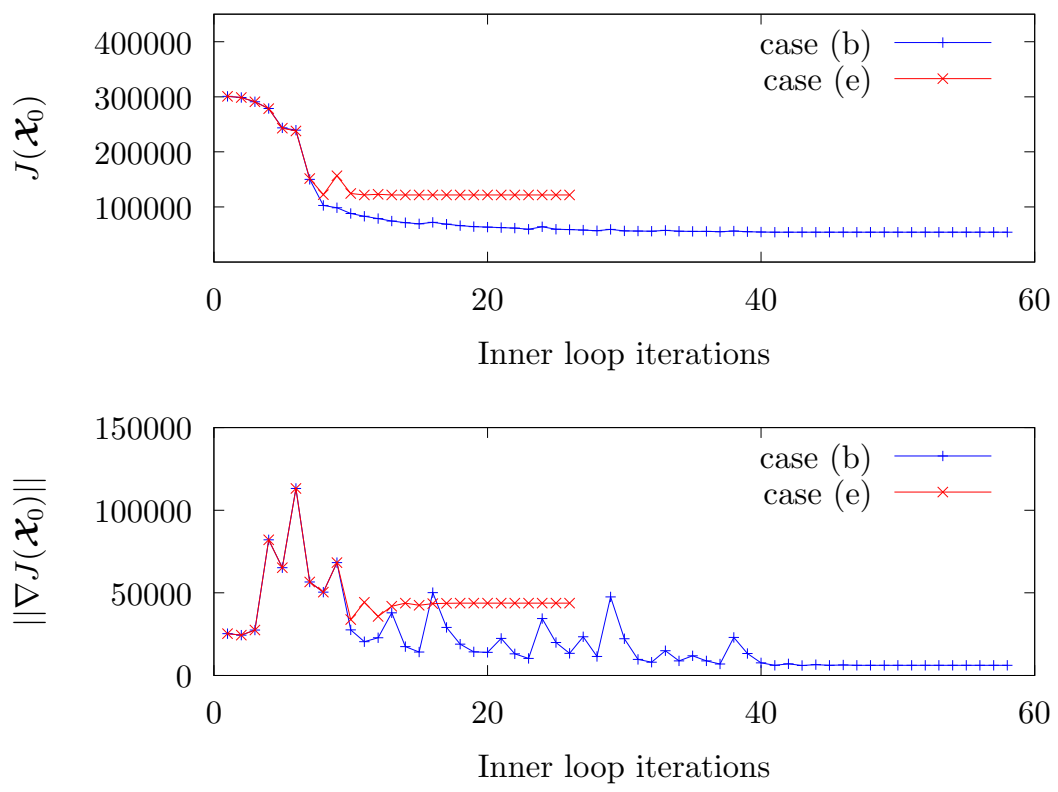

Figure 15: Evolution of the cost function $J\left(\mathcal{X}_{0}\right)$ and its gradient $\left\|\nabla J\left(\mathcal{X}_{0}\right)\right\|$ for the assimilation as a function of inner loop iterations for cases (b) and (e).

this could be improved by estimating the observation covariance matrix using a similar methodology and by weighting appropriately the various contributors to the cost function.

\subsection{Sliding window optimisation}

A well-known drawback of VDA is the inability to assimilation observations over a long assimilation time-window. In meteorological applications, the temporal range of the assimilation window is estimated as the inverse of the first Lyapunov exponent. This indicates the time-period within which a reliable forecast can be made. A time-window larger than this estimate could result in the unstable modes of the dynamics diverging quickly from the observations. This limitation is why weather forecasting is restricted to a maximum prediction of ten-days. As we shift focus to turbulent fluid flows, the time-domain associated with the much finer scales of motion is significantly smaller. In our $4 \mathrm{D}$-Var 

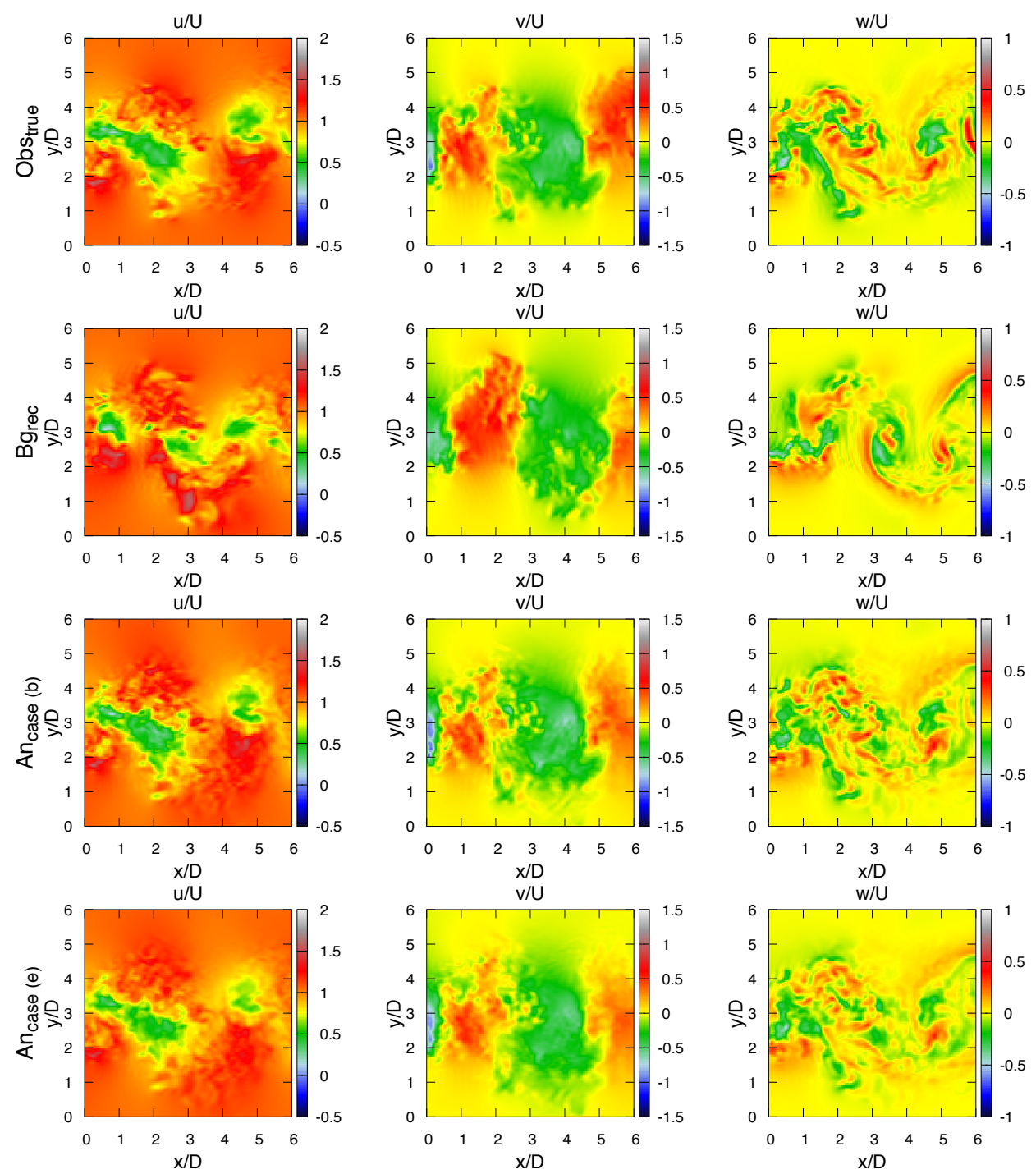

Figure 16: Velocity snapshots at the beginning of the assimilation window $\left(t_{1}^{\prime}\right)$ in the plane $z=1.31 \mathrm{D}$. From left to right: streamwise, lateral, and spanwise fields. From top to bottom: $O b s_{\text {true }}, B g_{\text {rec }}, A n_{\text {case (b) }}$, and $A n_{\text {case (e) }}$

algorithm, we restrict ourselves to only 100 time-steps for the assimilation window. This value has been empirically set. Estimation of the first Lyapunov exponent could lead to optimal size. However, such an estimation is very time consuming. This limitation is also inherent to SDA algorithms which requires 

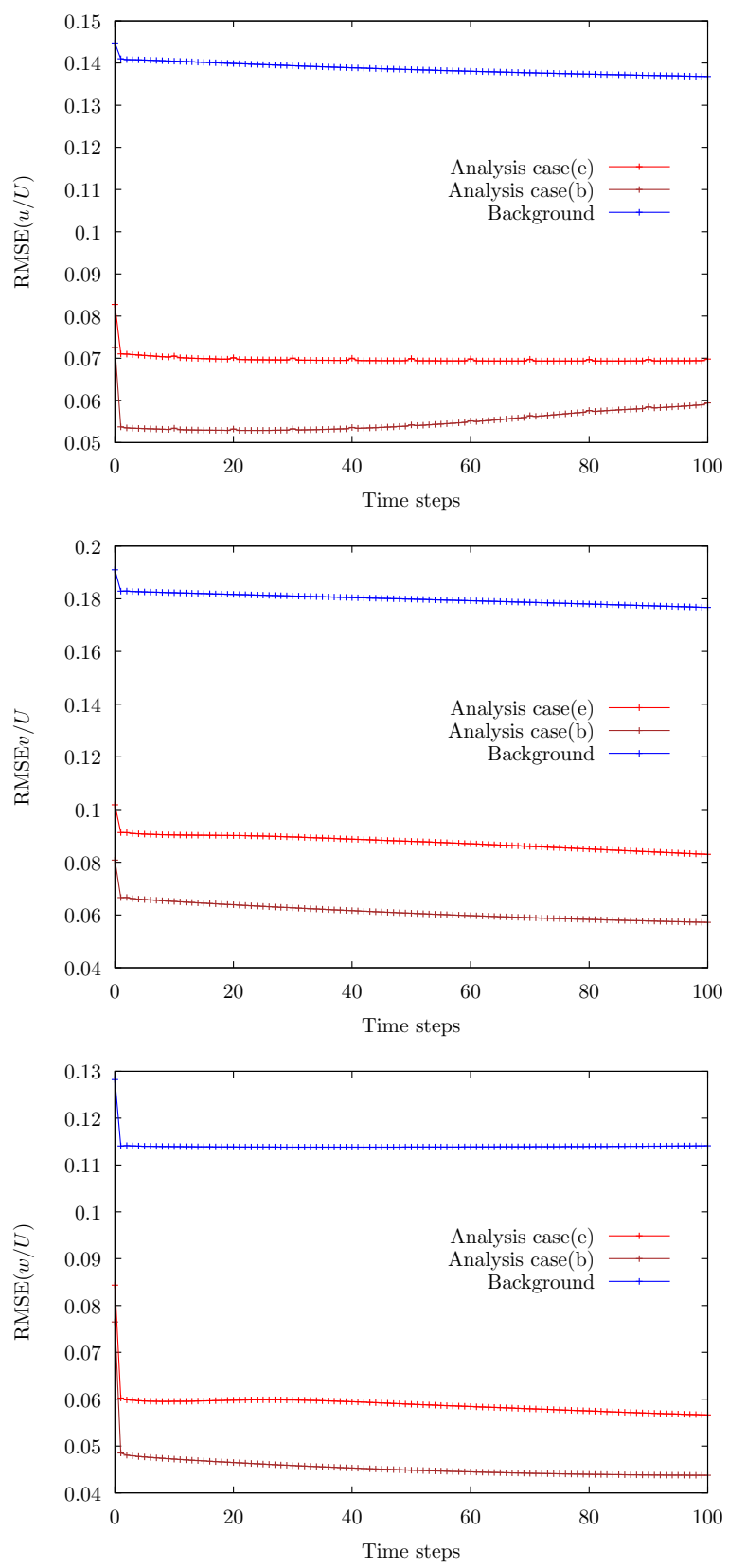

Figure 17: Evolution of the RMSE along time of the three velocity components between the true observations and the assimilation components, namely background, observation, and analysis for case (d). 
larger ensembles for larger time-domains with increased observations leading to computational and memory difficulties.

In VDA, an innovative way to extend the time-period of assimilation is to use the concept of sliding windows [15, 16. In this methodology, the 4D-Var local grid computer.

In this section, we have performed the sliding window approach for one vortex shedding cycle, as a proof of concept, to show its capabilities for long term VDA. Three runs are performed: first with the true background and true obser630

vations ( $D A_{\text {ideal }}$ ), second with a reconstructed background and true observations $\left(D A_{b g}\right)$, and the third with reconstructed background and reconstructed 
observations $\left(D A_{r e c}\right)$. The velocity contours at the end of the final sliding window, corresponding to the 1700th time-step, is shown for the three cases along with the true and reconstructed observations at this instance in figure 635 18. With true observations, the assimilation, despite the background condition, captures the flow field accurately with respect to the observations. For $D A_{r e c}$, the velocity contours appears to be at an intermediate state between the true observations and the reconstructed observations.

The lateral profiles of statistical quantities at three streamwise locations $(X=0.5 D ; 1 D ; 2 D$ in the assimilation domain) are presented in figures 19.20 - the values have been translated for concise representation. Two additional direct simulations are run in the assimilation domain for one vortex shedding with the true background $\left(B g_{\text {true }}\right)$ and the reconstructed background $\left(B g_{r e c}\right)$ to compared with the DA results. The profiles are matched with the DNS reference 645 from [8].

All simulations match well with the reference for the mean streamwise profiles. The use of the sliding window provides additional time-steps for statistical averaging. Thus, the DA profiles are smoother and more converged than the direct profiles. The use of the averaged SO observations provides less turbulent, smooth flow fields. This leads to a smoother profile for $D A_{r e c}$ as compared to the other cases. The mean spanwise profile suggests that one vortex shedding is clearly not enough for statistical convergence for this quantity. It is interesting to note that when the observations provided for DA are turbulent, as is the case for $D A_{\text {ideal }}$ and $D A_{b g}$, the convergence or smoothness of the profiles are reduced as compared $D A_{r e c}$. The use of the averaging SO observations leads to significantly smoother profiles.

This does have an associated disadvantage with respect to the turbulent fluctuation profiles in figure 20. A clear under prediction of statistics is observed for $D A_{r e c}$ - a result of the smooth observations used. However, even in the fluctuation statistics, the $D A_{r e c}$ has the most converged statistics that matches best, in profile, with the reference albeit with lower magnitude. These results are encouraging towards the capability of $4 \mathrm{D}$-Var to extend to larger 

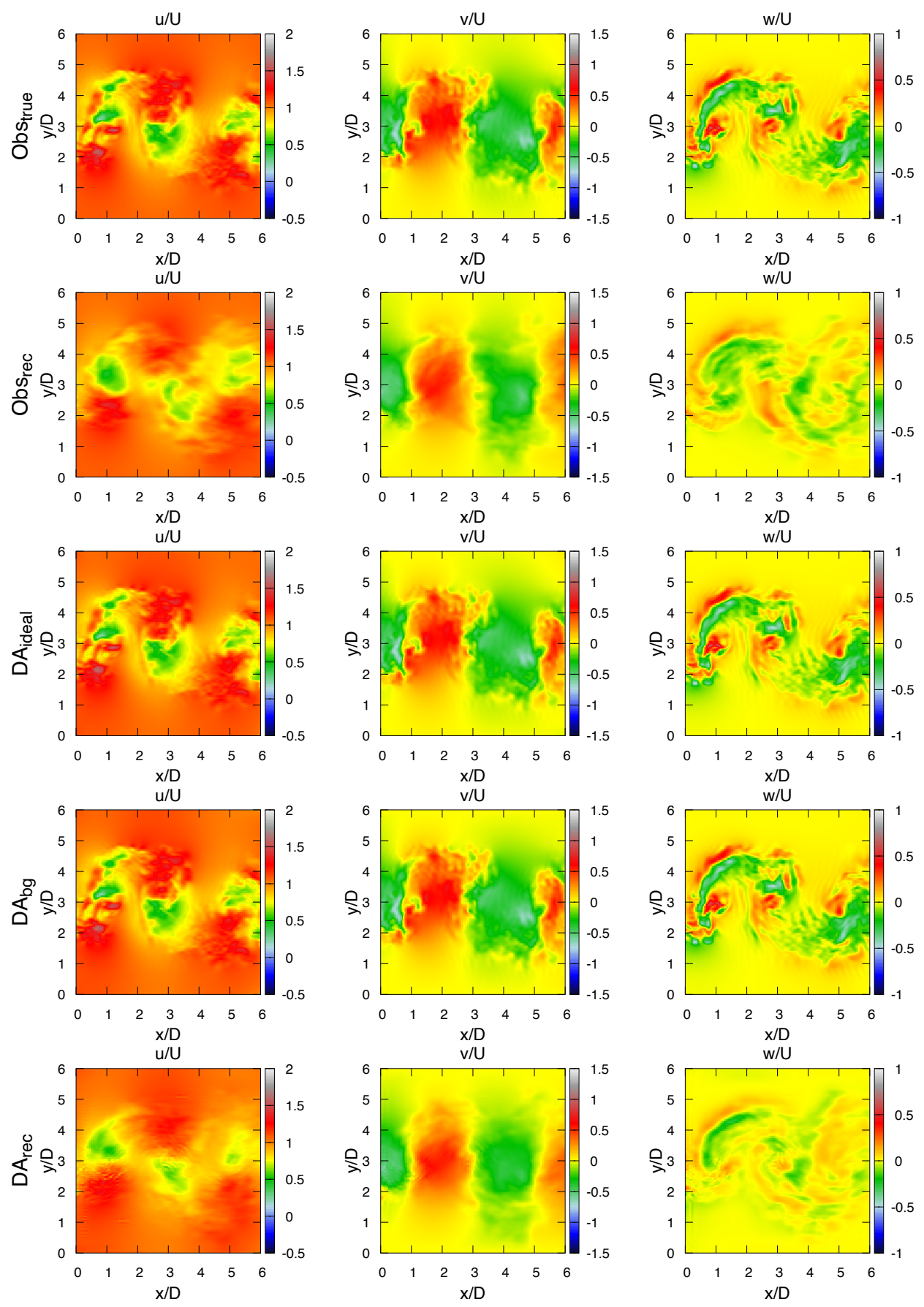

Figure 18: Velocity snapshots at the end of the final sliding window $\left(t_{f}^{\prime}=1700\right)$ in the plane $z=1.31 \mathrm{D}$. From left to right: streamwise, lateral, and spanwise fields. From top to bottom: $O b s_{\text {true }}, O b s_{r e c}$, Analysis for $D A_{\text {ideal }}, D A_{b g}$ and $D A_{r e c}$ sliding window cases. 


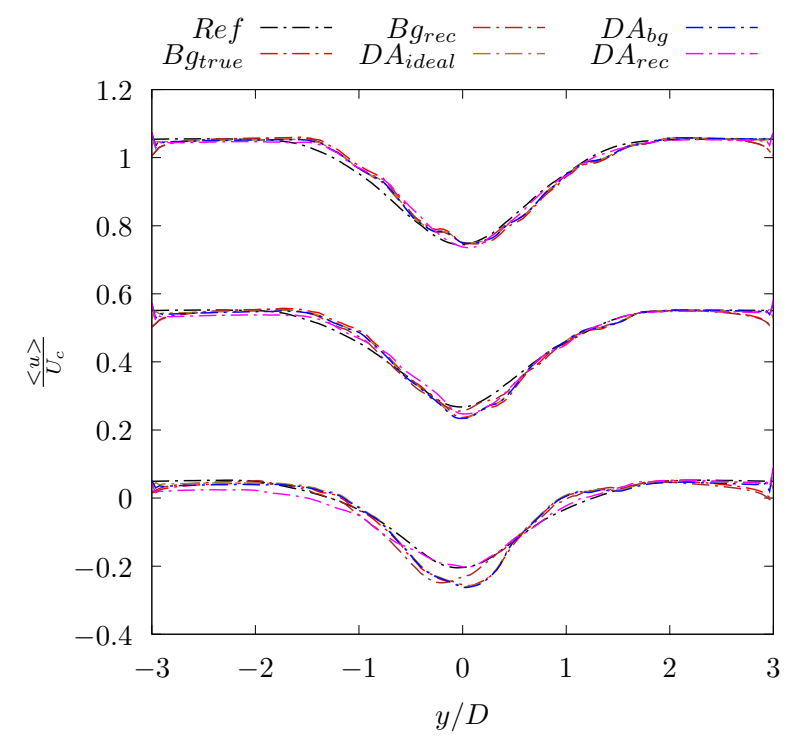

(a) $\langle u>$

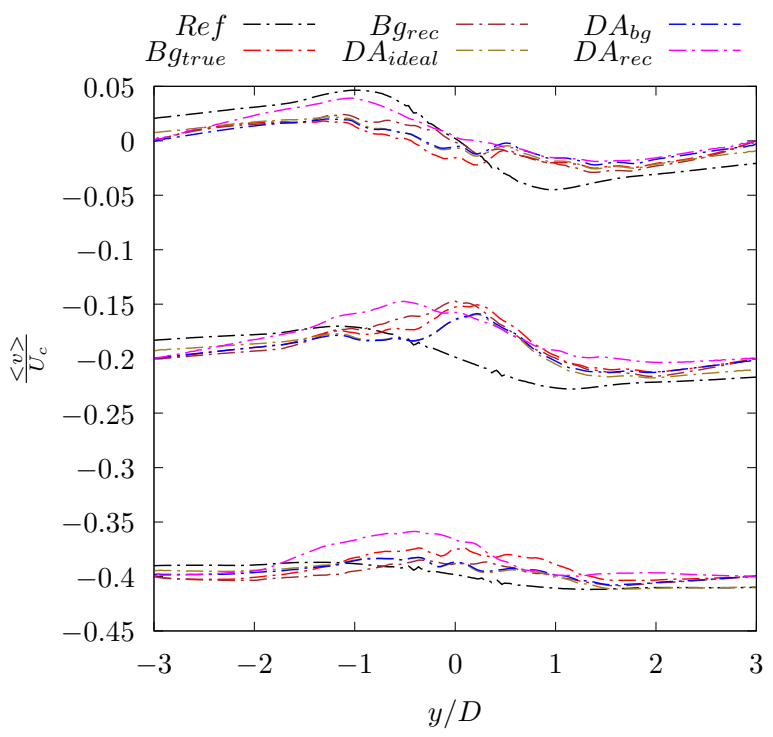

(b) $\langle v>$

Figure 19: Mean streamwise (a) and lateral (b) velocity profile along $y$, averaged along the spanwise $(z)$ direction.

assimilation time-domain with the help of sliding windows. It must be noted that the associated computational cost and time for performing sliding window 


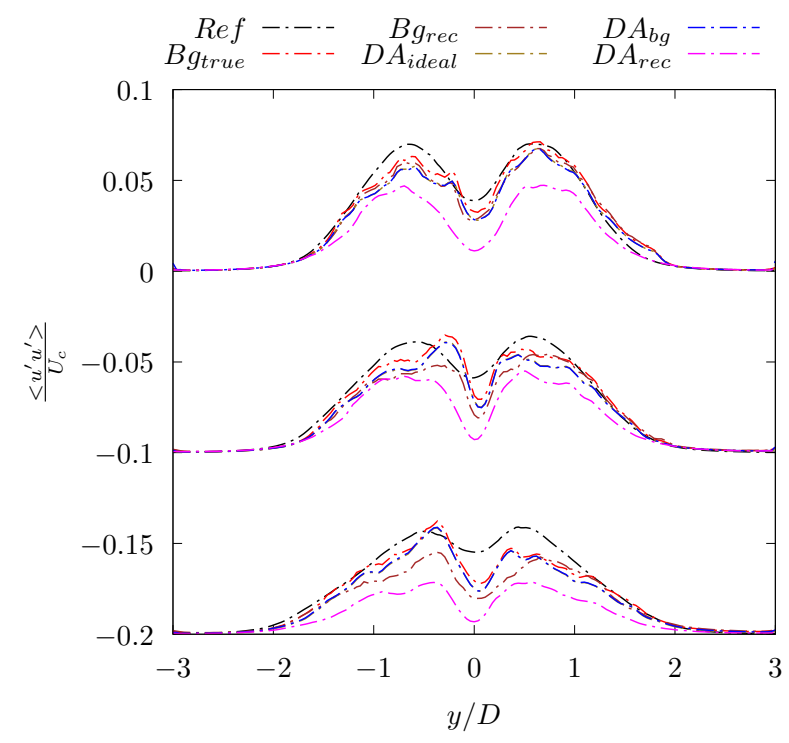

(a) $\left\langle u^{\prime} u^{\prime}>\right.$

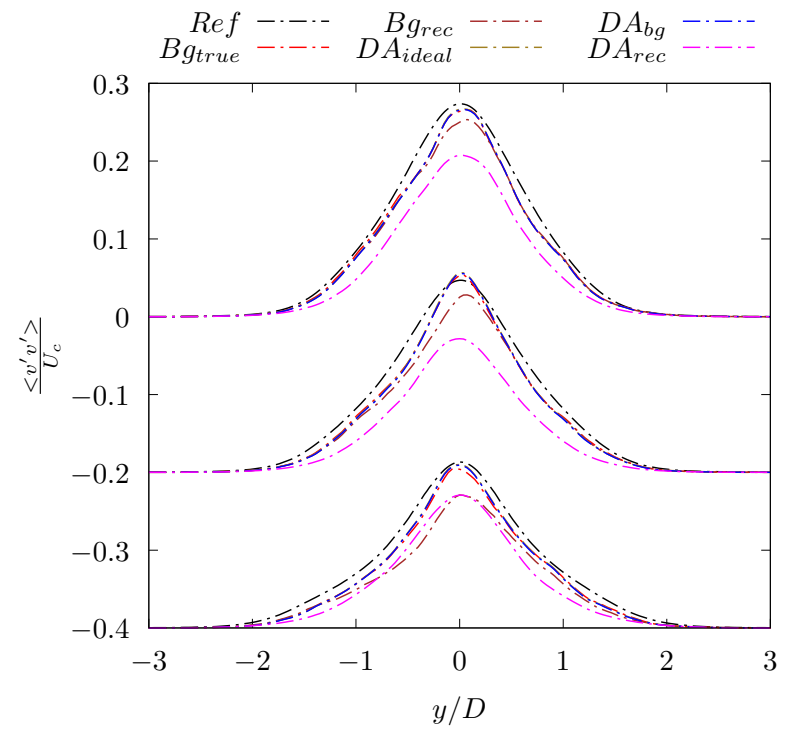

(b) $\left\langle v^{\prime} v^{\prime}\right\rangle$

Figure 20: Fluctuating streamwise (a) and lateral (b) velocity profile along $y$, averaged along the spanwise $(z)$ direction.

assimilation needs to be considered. 


\section{Error model optimisation}

By introducing the error model coefficient $\left(C_{s p}\right.$ in eq. $\left.32 p\right)$ as a control parameter $(\boldsymbol{\vartheta})$ in the variational assimilation algorithm, it can be optimised along with the initial velocity field maps. Estimation of an error model coefficient

To the extent of the authors' knowledge, this is the first attempt at analysing the capability of a VDA algorithm for estimating an error model coefficient for a turbulent flow. As explained in $\$ 2$, this strategy provides a compromise solution between a strong assimilation constraint (case explored in $\$ 4$ and a weak-constraint assimilation.

An initial estimate (equivalent to the background condition) for the coefficient is provided using eq. (32) - for a uniform mesh, this results in a constant coefficient estimate in time and space. Let us note that this value corresponds to the value used for the reference simulation (but with a stretched mesh and known boundary conditions). The optimised coefficient is enforced to be constant in time but allowed to vary in space. A penalty term is considered (see cost function eq. (39) below) on the coefficient to ensure it does not vary far from the background value provided. This is important for multiple reasons:

- The coefficient defines the amount of dissipation brought in by the model and the stability of the flow is predicated on this;

- The contribution to the cost function of the coefficient is only through the deviation from the initial estimate provided and this is multiple orders of magnitude smaller than the observation error. Thus, the algorithm tends to modify freely the coefficient in order to reduce the cost function in lieu of the initial/inlet condition.

The cost function including the additional control on the coefficient is given as,

$$
J(\boldsymbol{\eta}, \vartheta)=\frac{1}{2}\|\boldsymbol{\eta}\|_{\boldsymbol{B}^{-1}}^{2}+\frac{1}{2}\|\vartheta\|_{\boldsymbol{B}_{c}^{-1}}^{2}+\frac{1}{2} \int_{t_{0}}^{t_{f}}\left\|\mathbb{H}\left(\boldsymbol{\mathcal { X }}_{t}\right)-\mathcal{Y}(t)\right\|_{\boldsymbol{R}^{-1}}^{2} d t
$$


where $\vartheta=C_{0}-C_{b}$ is the difference between the coefficient estimate and the background value given by eq. 32 , and $\boldsymbol{B}_{c}^{-1}$ is the corresponding covariance which is set to Identity. The gradient, using the adjoint approach, is defined as,

$$
\begin{gathered}
\frac{\partial J}{\partial \boldsymbol{\eta}}=-\boldsymbol{\lambda}_{t_{0}}+\boldsymbol{B}^{-1} \delta \boldsymbol{\mathcal { X }}_{0}, \\
\frac{\partial J}{\partial \vartheta}=-\boldsymbol{\lambda}_{t_{0}}+\boldsymbol{B}_{c}^{-1} \vartheta+\left(\partial_{\vartheta} \mathbb{M}\right)^{*} \boldsymbol{\lambda} .
\end{gathered}
$$

where $\left(\partial_{\vartheta} \mathbb{M}\right)^{*}$ refers to the adjoint dynamic model with respect to the coefficient formulated using TAPENADE, the AD tool. It is interesting to note that due to the linear nature of the coefficient in the dynamical model (see eq. (31)), the tangent dynamical model with respect to the coefficient is the model itself without the coefficient.

An assimilation study (case (f)) is performed with reconstructed observations and a reconstructed background condition emulating experimental measurements with a fully-defined inverse of the background covariance matrix. This case corresponds thus to the most finalised assimilation strategy with experimentally realistic measurements and an error model calibrated from these data. The coefficient is allowed to vary in space while it is assumed constant in time. The resultant analysis trajectory is similar to that of case (d) and is not shown here to avoid repetition. Figure 21 presents the 2D contour map of the initial and optimised model coefficient. The optimised coefficient varies in space and is in general stronger than the initial condition obtained from the model equation implying higher dissipation in such regions. These regions of higher value matches well with the locations of strong vorticity of the flow (see figure 22). Such regions of high turbulence imply active sub-grid scales and thus require a stronger contribution of the model characterised by the larger coefficient value.

The validity of the optimised coefficient is supported by the RMSE evolution in figure 23 where two identical cases with and without coefficient optimisation are compared. By optimising the coefficient, a lower RMSE is obtained for all 

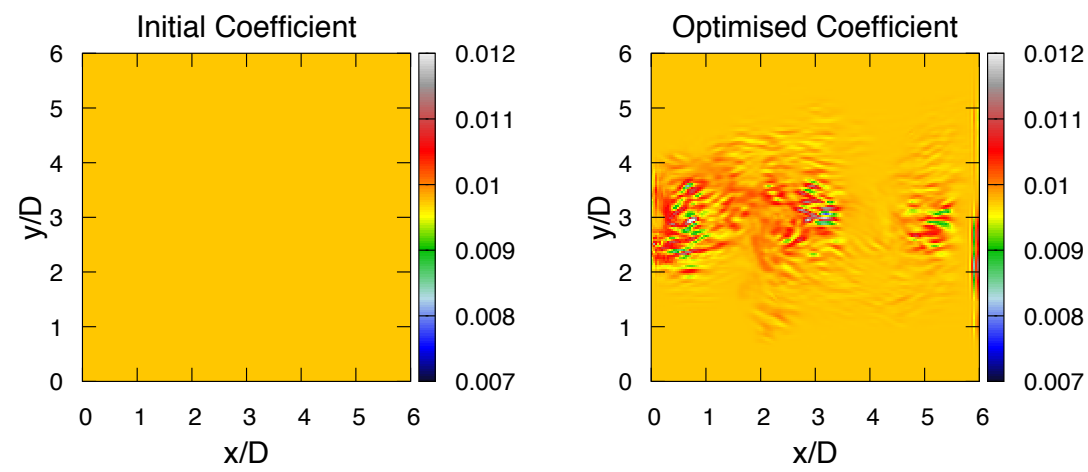

Figure 21: LES model coefficient contour map in the streamwise plane $z=1.31 \mathrm{D}$ for case (f). From left to right: background, and analysis.

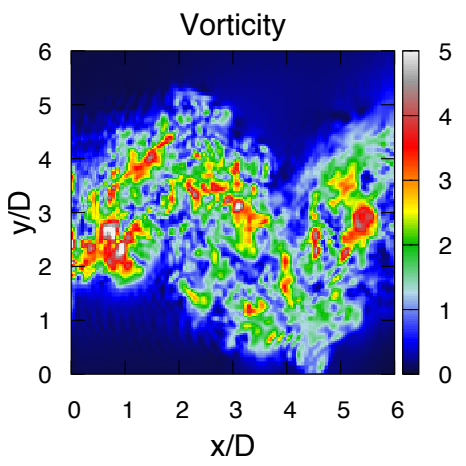

Figure 22: Instantaneous flow vorticity in the streamwise plane $z=1.31 \mathrm{D}$ calculated from the true observations.

three velocity components than with a fixed coefficient despite both requiring

roughly the same number of iterations. In addition, this error gap between the two cases appears to widen as we evolve along time with improved prediction with the optimised coefficient. This suggests that the optimised coefficient is a better representation of the dynamical evolution of the flow taking into account the small-scale contributions. Thus, an evolution in time beyond the assimilation window should further improve the prediction than with a non-optimised coefficient. A sliding window algorithm with coefficient optimisation can easily be envisioned, however, it has not been attempted due to the high computa- 
tional cost requirements. These preliminary results are very interesting and shows the capability of the proposed assimilation scheme to go towards error model parametrisation, a concept that has been at the forefront of research over many decades [3, 4, 21. Let us stress again that these results are obtained here without any modelling of the obstacle. Only a symmetry of the obstacle is exploited in the reconstruction method. This technique provides rough volumetric observations and is associated with a covariance matrix that strongly penalises observations far from the observed planes. As a consequence, this technique could probably also be used for non-symmetric obstacles.

\section{Conclusions}

This article presents a new variational data assimilation approach, termed as 4D-Var, building on the work of Gronskis et al. 20] for assimilation of 3D, unsteady, incompressible turbulent flows. The LU turbulence model of Mémin [29] was coupled with VDA principles to take into account errors in the dynamics of the assimilated model. Such a strategy enables us to consider a variational assimilation strategy which is a compromise between a strong constraint assimilation without error, and a weakly constrained assimilation with a non-informative Gaussian error model.

The 4D-Var algorithm has been successfully applied to turbulent wake behind a circular cylinder at a Reynolds number of 3900 in the transitional regime of cylinder wake flow. A parametric study was performed on three crucial inputs for the 4D-Var algorithm, i.e. the background condition, the observation data-sets, and the background covariance matrix to conclude the following: A physically meaningful background improved the accuracy of the assimilation at lower cost than an inaccurate estimate of the background. The quality of the observation data-set was directly correlated to algorithm stability. Analysis trajectories incorporating interpolated observations were seen to be unstable when non-linearly evolved in time. Observations reconstructed using the SO methodology were better suited giving stable analysis velocity fields. A fully-defined 

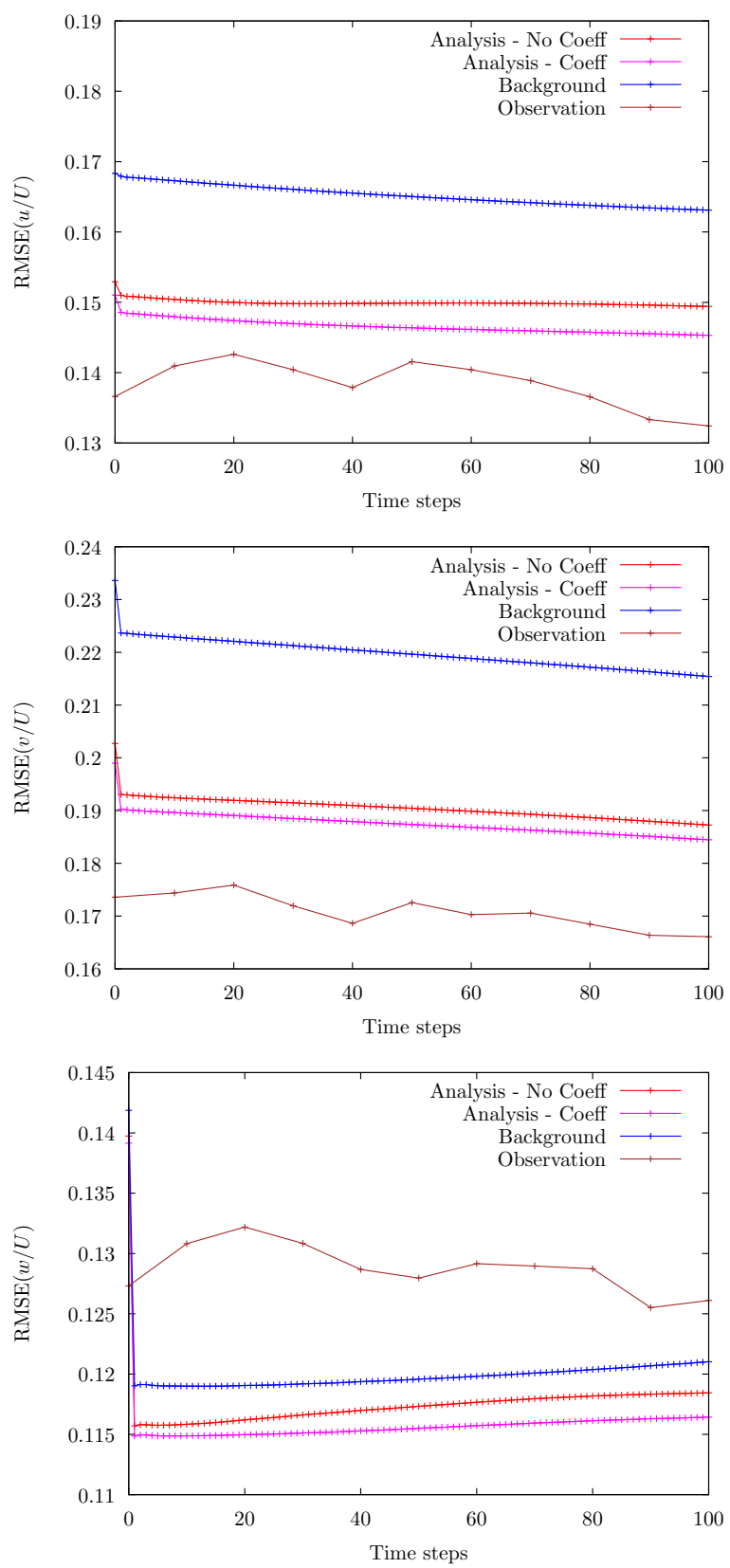

Figure 23: Evolution of the RMSE along time of the three velocity components between the true observations and the assimilation components, namely background, observation, and analysis for assimilation with and without coefficient optimisation. 
inverse of the background covariance matrix provides faster convergence requiring only $\sim 50 \%$ of the iterations but with an associated loss of accuracy $15-20 \%$ at the beginning of the assimilation window in comparison with results obtained for an inverse background covariance matrix set to Identity.

An interesting avenue of research opened by combining an error model, representing turbulent small-scale flow structures, with an optimisation approach is the ability to tune model contribution predicated on observations. For the $\mathrm{LU}$ model, this tuning is introduced via the model coefficient, $C_{s p}$, which is implemented as a control parameter in the 4D-Var algorithm and optimised along with the initial condition. The tuned coefficient was observed to be stronger in regions corresponding to high vorticity, i.e. regions of high turbulence and thus stronger sub-grid scale activity, i.e. larger model error. The RMSE for the optimal velocity prediction with the tuned coefficient is lower than when the model coefficient is not optimised. This proposed assimilation strategy coupled with the LU modelling allows us, for the first time to the authors knowledge, to consider the reconstruction of a 3D turbulent flow without modelling explicitly the obstacle geometry together with an explicit control of the error model. In this work, we have shown that parameter estimation is possible with VDA and provides good results despite the requirement of an additional adjoint with respect to the parameter.

Future work would focus on the application of the algorithm to experimental data-sets such as cross-plane PIV measurements or volumetric PIV measurements. The results presented here with reconstructed observations emulating experimental data were encouraging towards assimilation of such experimental observations. The control of boundary conditions as parameters in the $4 \mathrm{D}$-Var algorithm is another interesting avenue of research that needs exploration. With the current algorithm, small discontinuities can be observed at the outlet condition for certain parameters, for example with the optimised model coefficient. By implementing the outlet condition as a control parameters, a better estimate could probably be obtained at the outlet. 


\section{References}

\section{References}

[1] Artana, G., Cammilleri, A., Carlier, J., Mémin, E., 2012. Strong and weak constraint variational assimilations for reduced order fluid flow modeling. J. Comput. Phys. 231 (8), 3264-3288.

[2] Bergthórsson, P., Döös, B. R., 1955. Numerical Weather Map Analysis. Tellus 7 (3), 329-340.

[3] Cardinali, C., Žagar, N., Radnoti, G., Buizza, R., Sep. 2014. Representing model error in ensemble data assimilation. Nonlinear Processes in Geophysics 21 (5), 971-985.

[4] Carrassi, A., Vannitsem, S., Nicolis, C., Jul. 2008. Model error and sequential data assimilation: A deterministic formulation. Quarterly Journal of the Royal Meteorological Society 134 (634), 1297-1313.

[5] Chandramouli, P., 2018. Turbulent complex flows reconstruction via data assimilation in large eddy models. Scientific, Université de Rennes 1, Rennes.

[6] Chandramouli, P., Mémin, E., Chapron, B., Laizet, S., Heitz, D., ???? Deciphering the role of small-scale inhomogeneity on flow structuration: a stochastic approach. Submitted to Science.

[7] Chandramouli, P., Memin, E., Heitz, D., Fiabane, L., 2019. Fast 3d flow reconstructions from 2d cross-plane observations. Experiments in Fluids 60,30 .

[8] Chandramouli, P., Memin, E., Heitz, D., Laizet, S., 2018. Coarse large-eddy simulations in a transitional wake flow with flow models under location uncertainty. Comput. Fluids 168, 170-189. 
[9] Chapron, B., Derian, P., Mémin, E., Resseguier, V., 2018. Large scale flows under location uncertainty: a consistent stochastic framework. Q. J. R. Meteorolog. Soc. 144 (710), 251-260.

[10] Cressman, G. P., 1959. An operational objective analysis system. Mon. Weather Rev. 87 (10), 367-374.

[11] D'adamo, J., Papadakis, N., Mémin, E., Artana, G., 2007. Variational assimilation of POD low-order dynamical systems. J. Turbul. 8, N9.

[12] Elsinga, G. E., Scarano, F., Wieneke, B., van Oudheusden, B. W., 2006. Tomographic particle image velocimetry. Exp. Fluids 41 (6), 933-947.

[13] Evensen, G., 1994. Sequential data assimilation with a nonlinear quasigeostrophic model using Monte Carlo methods to forecast error statistics. J. Geophys. Res. Oceans 99 (C5), 10143-10162.

[14] Evensen, G., 2009. The ensemble Kalman filter for combined state and parameter estimation. IEEE Control Systems 29 (3).

[15] Fang, P., Bock, Y., 1998. Sliding window procedure for super near real-time continuous GPS water vapor estimation using predicted orbits. Annales Geophysicae 16 (Suppl. I).

[16] Foster, J., Bevis, M., Businger, S., Jun. 2005. GPS Meteorology: SlidingWindow Analysis*. Journal of Atmospheric and Oceanic Technology 22 (6), 687-695.

[17] Foures, D. P. G., Dovetta, N., Sipp, D., Schmid, P. J., 2014. A dataassimilation method for Reynolds-averaged Navier-Stokes-driven mean flow reconstruction. J. Fluid Mech. 759, 404-431.

830 [18] Gautier, R., Laizet, S., Lamballais, E., 2014. A DNS study of jet control with microjets using an immersed boundary method. Int. J. Comput. Fluid Dyn. 28 (6-10), 393-410. 
[19] Gordon, N. J., Salmond, D. J., Smith, A. F. M., 1993. Novel approach to nonlinear/non-Gaussian Bayesian state estimation. IEE Proceedings F - Radar and Signal Processing 140 (2), 107.

[20] Gronskis, A., Heitz, D., Mémin, E., 2013. Inflow and initial conditions for direct numerical simulation based on adjoint data assimilation. J. Comput. Phys. 242, 480-497.

[21] Harlim, J., 2017. Model error in data assimilation. In: Nonlinear and Stochastic Climate Dynamics. Cambridge University Press.

[22] Hascoët, L., Pascual, V., 2004. TAPENADE 2.1 user's guide. Tech. rep.

[23] Kadri Harouna, S., Mémin, E., 2017. Stochastic representation of the Reynolds transport theorem: revisiting large-scale modeling. Comput. Fluids $156,456-469$.

[24] Kim, J., Bodony, D., Freund, J., Jun. 2010. A High-Order, Overset-Mesh Algorithm for Adjoint-Based Optimization for Aeroacoustics Control. In: 16th AIAA/CEAS Aeroacoustics Conference. American Institute of Aeronautics and Astronautics, Stockholm, Sweden.

[25] Laizet, S., Lamballais, E., 2009. High-order compact schemes for incompressible flows: A simple and efficient method with quasi-spectral accuracy. J. Comput. Phys. 228 (16), 5989-6015.

[26] Le Dimet, F.-X., Talagrand, O., 1986. Variational algorithms for analysis and assimilation of meteorological observations: theoretical aspects. Tellus A $38(2), 97-110$.

855 [27] Lions, J., 1971. Optimal control of systems governed by partial differential equations problèmes aux limites. Berlin: Springer, 407.

[28] Meldi, M., Poux, A., 2017. A reduced order model based on Kalman filtering for sequential data assimilation of turbulent flows. J. Comput. Phys. 347, 207-234. 
[37] Resseguier, V., Mémin, E., Chapron, B., 2017. Geophysical flows under location uncertainty, Part III SQG and frontal dynamics under strong turbulence conditions. Geophys. Astro. Fluid 111 (3), 209-227.

[38] Sasaki, Y., 1958. An Objective Analysis Based on the Variational Method.

[29] Mémin, E., 2014. Fluid flow dynamics under location uncertainty. Geophys. Astro. Fluid 108 (2), 119-146.

[30] Mons, V., Chassaing, J.-C., Gomez, T., Sagaut, P., 2016. Reconstruction of unsteady viscous flows using data assimilation schemes. J. Comput. Phys. $316,255-280$.

[31] Mons, V., Chassaing, J.-C., Sagaut, P., Jul. 2017. Optimal sensor placement for variational data assimilation of unsteady flows past a rotationally oscillating cylinder. Journal of Fluid Mechanics 823, 230-277.

[32] Nocedal, J., 1980. Updating quasi-Newton matrices with limited storage. Math. Comput. 35 (151), 773-782.

[33] Papadakis, N., Mémin, E., Cuzol, A., Gengembre, N., 2010. Data assimilation with the weighted ensemble Kalman filter. Tellus A 62 (5), 673-697.

[34] Parnaudeau, P., Carlier, J., Heitz, D., Lamballais, E., 2008. Experimental and numerical studies of the flow over a circular cylinder at Reynolds number 3900. Phys. Fluids 20 (8), 085101.

[35] Resseguier, V., Mémin, E., Chapron, B., 2017. Geophysical flows under location uncertainty, Part I Random transport and general models. Geophys. Astro. Fluid 111 (3), 149-176.

[36] Resseguier, V., Mémin, E., Chapron, B., 2017. Geophysical flows under location uncertainty, Part II Quasi-geostrophy and efficient ensemble spreading. Geophys. Astro. Fluid 111 (3), 177-208.

J. Meteorolog. Soc. Jpn. Ser. II 36 (3), 77-88. 
[39] Sasaki, Y., 1970. Some basic formalisms in numerical variational analysis. Mon. Weather Rev. 98 (12), 875-883.

[40] Suzuki, T., Ji, H., Yamamoto, F., Jul. 2010. Instability waves in a lowReynolds-number planar jet investigated with hybrid simulation combining particle tracking velocimetry and direct numerical simulation. Journal of Fluid Mechanics 655, 344-379.

[41] Tissot, G., Cordier, L., Benard, N., Noack, B. R., 2014. Model reduction using dynamic mode decomposition. C. R. Mécanique 342 (6-7), 410-416.

[42] Yang, Y., Mémin, E., 2017. High-resolution data assimilation through stochastic subgrid tensor and parameter estimation from 4denvar. Tellus A $69,1-24$.

[43] Yang, Y., Robinson, C., Heitz, D., Mémin, E., 2015. Enhanced ensemblebased 4dvar scheme for data assimilation. Comput. Fluids 115, 201-210.

\section{Appendix A. Interpolation method for Obsinter}

The interpolation method used for obtained $O b s_{\text {inter }}$ in 4.4 .2 is as follows,

$$
\begin{gathered}
w_{1}=\exp \left(-\left(x-x_{0}\right)^{2} /(2 .)\right) \\
w_{2}=\exp \left(-\left(z-\left(\frac{L_{z}}{2}\right)\right)^{2} /(2 .)\right) \\
\boldsymbol{u}(x, y, z)=\left(\boldsymbol{u}\left(x_{0}, y, z\right) * w_{1}+\boldsymbol{u}\left(x, y, z_{0}\right) * w_{2}\right) /\left(w_{1}+w_{2}\right)
\end{gathered}
$$

$w_{1}$ defines the weight for the inlet plane velocity data while $w_{2}$ defines the weight for the XY plane in the middle of the spanwise domain.

\section{Appendix B. Calculation of $R_{3 D}^{-1}$ and $R_{P I V}^{-1}$}

The observation covariance matrix $\boldsymbol{R}_{3 D}^{-1}$ is implemented to be a function of space in order to account for boundary condition effects. A hyperbolic profile is used to reduce confidence in regions near the boundaries smoothly up to a 
user-defined minimum value $\left(\boldsymbol{R}_{\min }^{-1}\right)$. A user defined maximum $\left(\boldsymbol{R}_{\max }^{-1}\right)$ value is enforced in the middle of the domain. In the streamwise direction, due to the availability of measurements on the inlet plane, confidence is reduced only near the outlet boundary. The algorithm for defining the observation covariance matrix $\boldsymbol{R}_{3 D}^{-1}$ for the reference observations is mathematically expressed as,

$$
\begin{gathered}
\boldsymbol{R}_{3 D}^{-1}(x, y, z)=\frac{1}{2}\left(\tanh \left(-2.0\left(x-0.9 L_{x}\right)\right)+1\right) \\
\boldsymbol{R}_{3 D}^{-1}(x, y, z)=\boldsymbol{R}_{3 D}^{-1}(x, y, z) * \frac{1}{2}\left(\tanh \left(-2.0\left(y-\frac{L_{y}}{2}-0.5 L_{y}\right)\right)\right. \\
\boldsymbol{R}_{3 D}^{-1}(x, y, z)=\boldsymbol{R}_{3 D}^{-1}(x, y, z) * \frac{1}{2}\left(\tanh \left(-2.0\left(z-\frac{L_{z}}{2}-0.5 L_{z}\right)\right)\right. \\
\left.\quad-\tanh \left(-2.0\left(y-\frac{L_{y}}{2}+0.5 L_{y}\right)\right)\right) \\
\boldsymbol{R}_{3 D}^{-1}(x, y, z)=\boldsymbol{R}_{3 D}^{-1}(x, y, z) *\left(R_{\max }^{-1}-R_{\text {min }}^{-1}\right)+R_{\min }^{-1}
\end{gathered}
$$

The reconstructed or interpolated observations are not an exact representation of the reference field and thus, this needs to be reflected in the corresponding inverse of the observation covariance matrix $\left(\boldsymbol{R}_{P I V}^{-1}\right)$. Only the two observed planes command high confidence while the rest have minimal confidence defined up to a user defined minimum and maximum confidence $\left(\boldsymbol{R}_{\min }^{-1}\right.$ and $\boldsymbol{R}_{\max }^{-1}$ respectively). Thus, maximum confidence is given to the points falling on these two planes with an exponential decrease in confidence away from the observed 
planes. The covariance matrix is thus constructed as,

$$
\begin{array}{ll}
\boldsymbol{R}_{P I V}^{-1}(x, y, z)=\max \left(\exp \left(-\frac{x^{2}}{0.05}\right), \exp \left(-\frac{\left(z-0.5 L_{z}\right)^{2}}{0.05}\right), R_{m i n}^{-1}\right) \\
\boldsymbol{R}_{P I V}^{-1}(x, y, z)=\boldsymbol{R}_{P I V}^{-1}(x, y, z) * \exp \left(-\frac{\left(x-0.9 L_{x}\right)^{2}}{0.1}\right) & \forall x \geq 0.9 L_{x} \\
\boldsymbol{R}_{P I V}^{-1}(x, y, z)=\boldsymbol{R}_{P I V}^{-1}(x, y, z) * \exp \left(-\frac{\left(y-0.1 L_{y}\right)^{2}}{0.1}\right) & \forall y \leq 0.1 L_{y} \\
\boldsymbol{R}_{P I V}^{-1}(x, y, z)=\boldsymbol{R}_{P I V}^{-1}(x, y, z) * \exp \left(-\frac{\left(y-0.9 L_{y}\right)^{2}}{0.1}\right) & \forall y \geq 0.9 L_{y} \\
\boldsymbol{R}_{P I V}^{-1}(x, y, z)=\boldsymbol{R}_{P I V}^{-1}(x, y, z) * \exp \left(-\frac{\left(z-0.1 L_{z}\right)^{2}}{0.1}\right) & \forall z \leq 0.1 L_{z} \\
\boldsymbol{R}_{P I V}^{-1}(x, y, z)=\boldsymbol{R}_{P I V}^{-1}(x, y, z) * \exp \left(-\frac{\left(z-0.9 L_{z}\right)^{2}}{0.1}\right) & \forall z \geq 0.9 L_{z} \\
\boldsymbol{R}_{P I V}^{-1}(x, y, z)=\max \left(\boldsymbol{R}_{P I V}^{-1}(x, y, z), R_{\min }^{-1}\right) * R_{\max }^{-1} &
\end{array}
$$

On the mid streamwise plane and the inlet plane strong confidence is given taking into account boundary effect at the edges. Along the transverse plane, a steep exponential slope is enforced to reduce covariance as we move away from the two planes of true observation. 